bioRxiv preprint doi: https://doi.org/10.1101/2020.11.24.396002; this version posted April 1, 2021. The copyright holder for this preprint (which

was not certified by peer review) is the author/funder, who has granted bioRxiv a license to display the preprint in perpetuity. It is made available under aCC-BY 4.0 International license.

1

2

3

\title{
Initial ciliary assembly in Chlamydomonas requires Arp2/3 complex-dependent endocytosis
}

\author{
Brae M Bigge ${ }^{1,2}$, Nicholas E Rosenthal ${ }^{1,2}$, Prachee Avasthi ${ }^{1,2}$
}

${ }^{1}$ Biochemistry and Cell Biology Department, Geisel School of Medicine at Dartmouth College, Hanover, New Hampshire

${ }^{2}$ Anatomy and Cell Biology Department, University of Kansas Medical Center, Kansas City, Kansas 


\section{ABSTRACT} mechanisms of ciliary assembly also require the actin cytoskeleton. Here, we dissect subcellular roles of actin in ciliogenesis by focusing on actin networks nucleated by the Arp $2 / 3$ complex in the powerful ciliary model, Chlamydomonas. We find the Arp2/3 complex is required for the initial stages of ciliary assembly when protein and membrane are in high demand, but cannot yet be supplied from the Golgi complex. We provide evidence for Arp2/3 complex-dependent clathrin-mediated endocytosis of ciliary proteins, an increase in endocytic activity upon induction of ciliary growth, and relocalization of plasma membrane proteins to newly formed cilia. Our data support a new model of ciliary protein and membrane trafficking during early ciliogenesis whereby proteins previously targeted to the plasma membrane are reclaimed by Arp2/3 complex-dependent clathrin-mediated endocytosis for initial ciliary assembly. 


\section{INTRODUCTION}

The cilium of the unicellular, green alga Chlamydomonas reinhardtii has long been used as a model due to its structural and mechanistic conservation relative to the cilia of mammalian cells. Cilia consist primarily of microtubules that extend from the surface of the cell and are ensheathed in plasma membrane. Their assembly relies on microtubule dynamics and trafficking of protein and membrane (Nachury, Seeley, and Jin 2010), as well as intraflagellar transport (IFT), a motor-based transport system that moves tubulin and other cargo from the base of the cilium to the tip and back again (Pedersen and Rosenbaum 2008).

Although cilia are composed of microtubules and depend on them for assembly, the mechanisms governing ciliary maintenance and assembly extend to other cytoskeletal components, such as actin. In mammalian cells, actin disruption results in increased ciliary length and percentage of ciliated cells (Kim et al. 2010; Park et al. 2008). In fact, the microtubule organizing center of the cell, the centrosome, from which cilia are nucleated has been found to function as an actin organizer (Farina et al. 2016; Inoue et al. 2019). Further, in mammalian cells, when ciliogenesis is triggered by serum starvation, preciliary vesicles are trafficked to the centriole where they fuse to form a ciliary vesicle around the budding cilium. In the intracellular pathway of ciliogenesis, it has been shown that when Arp2/3 complex-branched actin is lost, depletion of preciliary vesicles at the centriole occurs due to problems with vesicle fusion, suggesting a role for branched actin in intracellular ciliogenesis (Wu, Chen, and Tang 2018). Further, actin itself has even been found within cilia, suggesting that actin is a key protein involved in ciliary maintenance and assembly (Kiesel et al. 2020).

Chlamydomonas cells are ideal for tackling the question of actin-dependent ciliary trafficking due to their lack of a cortical actin network and their ability to undergo more consistent and robust ciliogenesis than mammalian cells without requiring serum starvation. In Chlamydomonas, disruption of actin networks with Cytochalasin $\mathrm{D}(\mathrm{CytoD})$ resulted in shorter steady-state cilia (W. L. Dentler and Adams 1992) and disruption with Latrunculin B (LatB), which sequesters monomers leading to eventual filament depolymerization, resulted in shortened cilia and impaired regeneration (Avasthi et al. 2014; Jack et al. 2019). Chlamydomonas actin networks are required for accumulation of IFT machinery at the base of cilia and for entry of IFT material into cilia (Avasthi et al. 2014), as well as for trafficking of postGolgi vesicles to cilia, the synthesis of ciliary proteins, and the organization of the gating region at the base of cilia (Jack et al. 2019). Many key advances in our understanding of the relationship between cilia and actin have been discovered using Chlamydomonas, which is proving to be a useful model for studying the actin cytoskeleton and its ciliary functions.

The actin cytoskeleton of Chlamydomonas contains two actin genes: IDA5, a conventional actin with $91 \%$ sequence identity to human $\beta$-actin; and NAP1, a divergent actin that shares only $63 \%$ of its sequence with human $\beta$-actin (Hirono et al. 2003; Kato-Minoura et al. 1998). We consider NAP1 to be an actin-like protein as opposed to an actin related protein (ARP) because it has a higher sequency identity to actin than to conventional ARPs, and because it is able to functionally compensate for the conventional filamentous actin (Jack et al. 2019; M. Onishi et al. 2018; M. Onishi, Pringle, and Cross 2016; Masayuki Onishi et al. 2019). Under normal, vegetative conditions, the conventional IDA5 is the primary actin expressed, but when cells are treated with LatB, the LatB-insensitive NAP1 is upregulated (M. Onishi et al. 2018; M. Onishi, Pringle, and Cross 2016; Hirono et al. 2003). This separability of the two actins has led to the discovery that they can compensate for each other in ciliary maintenance and assembly (Jack et al. 2019). Studies of the role of actin in ciliary assembly have used global disruption by knocking out either one of the filamentous actins or acutely both, yet actin networks have diverse compositions and topologies that lead to specific subfunctions within cells.

Actin networks rely on the actin binding proteins that contribute to the formation, arrangement, and function of the network. One such actin binding protein is the Arp $2 / 3$ complex, 
which nucleates branched or dendritic actin networks by nucleating a daughter filament off the side of an existing mother filament. The dendritic networks nucleated by the Arp2/3 complex have been primarily found to be responsible for functions that involve membrane remodeling, for example lamellipodia and endocytosis (Campellone and Welch 2010). The Arp2/3 complex from most eukaryotes consists of seven subunits: Arp2, Arp3, and ARPC1-5 (Supplemental Figure 1) and each subunit plays a specific role of varying importance in the nucleation process. ARPC2 and ARPC4 form the core of the complex and the primary contacts with the mother filament, Arp2 and Arp3 serve as the first subunits of the daughter filament, and ARPC1 and ARPC3 play a role in nucleation but are not critical for branch formation (Robinson et al. 2001; Gournier et al. 2001). Each of these subunits are found in Chlamydomonas but have varying degrees of sequence homology compared with conventional Arp2/3 complexes (Supplemental Figure 1). Interestingly, the ARPC5 subunit has yet to be found in Chlamydomonas. ARPC5 is thought to be important for the association of ARPC1 to the complex, but a mammalian complex lacking ARPC5 and ARPC1 maintains some nucleating and branching activity and is able to cross-link actin normally (Gournier et al. 2001).

Here, using the chemical inhibitor CK-666 to inhibit the nucleating function of the Arp2/3 complex (Hetrick et al. 2013) and a genetic mutant of a critical Arp2/3 complex member, ARPC4 (Cheng et al. 2017; Li et al. 2019), we take a more delicate approach to investigating the role of actin in ciliary assembly by separating different actin networks into their subfunctions based on topology. Specifically, we probe the involvement of actin networks nucleated by the Arp2/3 complex in ciliary maintenance and assembly. This approach in these cells has allowed us to propose a new model implicating a subset of filamentous actin in redistribution of membrane and proteins for the initial stages of ciliogenesis.

\section{RESULTS}

Loss of Arp2/3 complex function inhibits normal regeneration and maintenance of cilia: We first investigated the requirement for the Arp2/3 complex in maintenance of cilia by treating cells with varying concentrations of CK-666, the inhibitor of the Arp2/3 complex, or the inactive control CK-689 $(250 \mu \mathrm{M})$ for 2 hours and measuring the effect on steady state ciliary length. Consistent with previous results (Avasthi et al. 2014), we found that treating cells with CK-666 decreased ciliary length, suggesting that the Arp2/3 complex is required for maintaining cilia (Figure 1A). We saw no changes in ciliary length with the inactive CK-689 (Figure 1A) or when arpc4 mutant cells lacking a functional Arp2/3 complex were treated with CK-666 (Supplemental Figure 2). Untreated arpc4 mutant cells recapitulate the CK-666 result and have a decreased ciliary length when compared with wild-type cells (Figure 1B) demonstrating through both chemical and genetic perturbation that the Arp2/3 complex is required for normal ciliary length maintenance.

Next, we probed the involvement of Arp2/3 complex-nucleated actin in the more complicated process of ciliary assembly where there is a high demand for protein and membrane both from pools already existing in the cell and from synthesis (Wingfield et al. 2017; Nachury, Seeley, and Jin 2010; Rohatgi and Snell 2010; Jack et al. 2019; Diener, Lupetti, and Rosenbaum 2015). Cells were deciliated by low pH shock and then allowed to synchronously regenerate cilia after being returned to normal $\mathrm{pH}$ (Paul A. Lefebvre 1995). We found that cells lacking a functional Arp2/3 complex were slow to regenerate their cilia, and two-thirds of cells did not regrow cilia at all (Figure 1C). Importantly, the initial steps are the slowest portion of assembly when existing protein and membrane are being incorporated into cilia.

This result is puzzling because the loss of Arp2/3 complex function, and therefore only a subset of actin filaments, results in a more dramatic phenotype than that of the nap1 mutants treated with LatB, which are lacking all filamentous actins (Jack et al. 2019). However, in the 
arpc4 mutant cells, a functional Arp2/3 complex never exists, and therefore, cells never have Arp2/3 complex-mediated actin networks. In nap1 mutant cells treated with LatB, the treatment begins shortly after deciliation causing an acute perturbation. Further, LatB functions by sequestering actin monomers to promote filament disassembly, and thus the effects may not be immediate (Spector et al. 1989). Therefore, it is likely that there is a brief window where actin filaments can assert their initial role in ciliary regeneration before being depolymerized. To avoid this, we began the LatB treatment in nap1 mutants 30 minutes before deciliation. This pretreatment allows us to observe what happens when actin is not present immediately after deciliation. (Figure 1D-E). In this case, we see decreased ciliary length and dramatically decreased percent ciliation, which is consistent with the arpc4 mutant results.

A

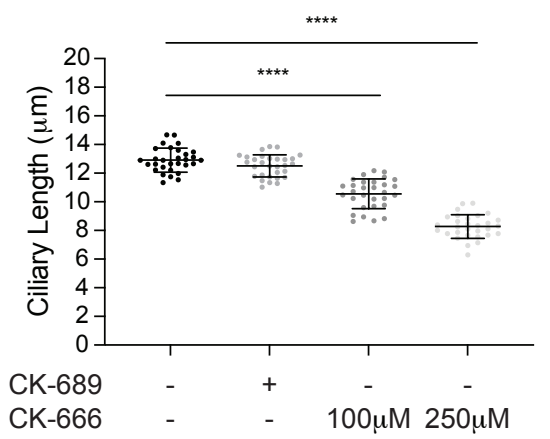

B

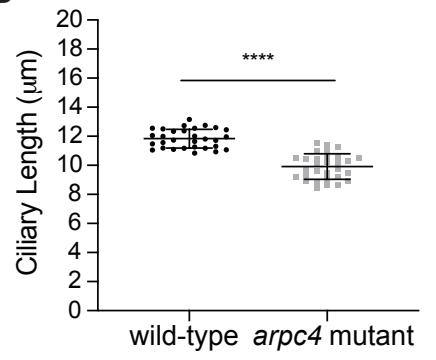

C

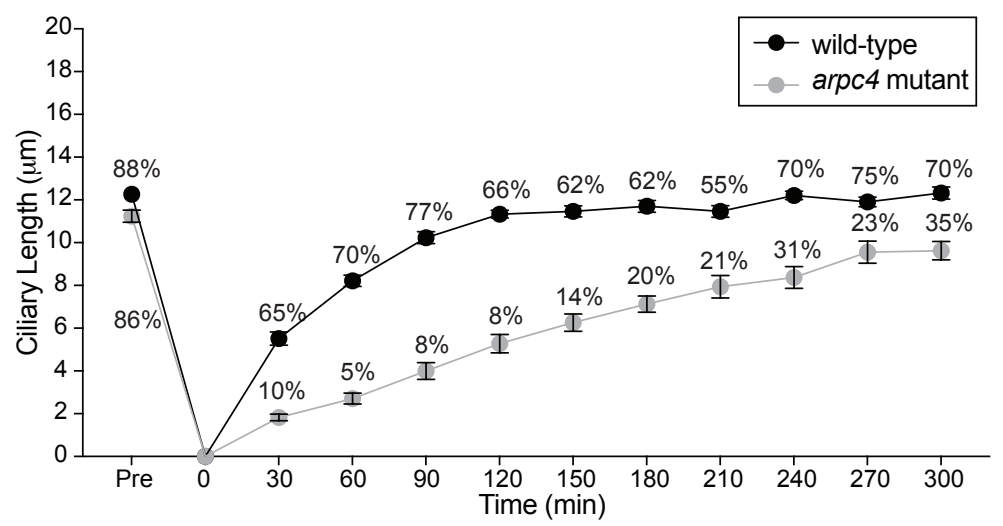

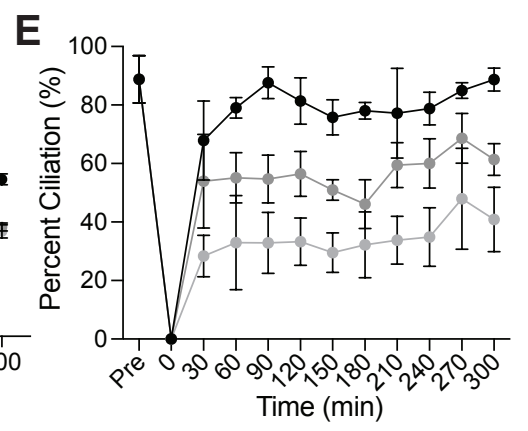

Figure 1. The Arp2/3 complex is required for normal ciliary maintenance and assembly. A) Wild-type cells were treated with $100 \mu \mathrm{M}$ or $250 \mu \mathrm{M}$ CK-666 or the inactive CK-689 for 2 hours. Cells were then imaged using a DIC microscope and cilia were measure in ImageJ. The mean is displayed with error bars representing standard deviation. $\mathrm{n}=30$ for each treatment in 3 separate experiments. $\mathrm{P}<0.0001$. B) Wild-type cells and arpc 4 mutant cells steady state cilia were also measured with no treatment. The mean is displayed with error bars representing standard deviation. $\mathrm{n}=30$ for each strain for 3 separate experiments. $\mathrm{P}<0.0001$. C) Wild-type cells and arpc4 mutant cells were deciliated using a $\mathrm{pH}$ shock and then allowed to regrow. The black line represents wild-type, while the grey line represents the arpc4 mutant. The numbers above or below each point show the percent ciliation for the wild-type and arpc4 mutant 
cells respectively. Means are displayed with error bars representing $95 \%$ confidence interval. $n=30$ for each strain and each time point in 3 separate experiments. For every time point except $0 \mathrm{~min}, \mathrm{P}<0.0001$ in terms of both length and percent ciliation. D) nap1 mutant cells were pre-treated with $10 \mu \mathrm{M}$ LatB for 30 minutes before deciliation or treated with LatB upon the return to neutral $\mathrm{pH}$ following deciliation. The black line represents untreated cells, while the light grey line represents cells treated with LatB following deciliation and the dark grey line represents cells pre-treated with LatB. Error bars represent standard deviation. $\mathrm{N}=3$ separate experiments. For every time point $\mathrm{P}>0.0001$ between $\mathrm{DMSO}$ and treated samples, except $30 \mathrm{~min}(10 \mu \mathrm{M}$ LatB) which is ns. Asterisks for the pretreatment are located below the graph, while asterisks for the post treatment are located above the line. E) Percent ciliation for the experiment in D. Line color is the same as D. Error bars represent standard deviation.

\section{The Arp2/3 complex is required for the incorporation of existing membrane and proteins for ciliary assembly:}

There are several distinct steps of ciliary assembly after severing, all of which require actin filaments. The first step requires that a pool of existing proteins and membrane are incorporated into cilia in an actin-dependent manner (Jack et al. 2019). As disruption of Arp2/3 complex-mediated actin networks results in slow initial ciliary assembly, when it is likely that existing protein is being incorporated, we questioned the involvement of Arp2/3 complexdependent actin networks in the incorporation of existing ciliary protein.

By treating cells with cycloheximide $(\mathrm{CHX})$, a protein synthesis inhibitor, we can eliminate the contribution of two steps in the process of ciliary assembly (Figure 2A) (Rosenbaum, Moulder, and Ringo 1969). Without protein synthesis, there is no trafficking or incorporation of new proteins. Therefore, any ciliary growth we see is due to the incorporation of the existing protein alone. Under normal conditions, cells that are deciliated and treated with cycloheximide typically grow cilia to about half-length, or $6 \mu \mathrm{m}$, within 2 hours (Figure 2B). We also saw cells growing to half-length after 2 hours when treated with the inactive CK-689 and cycloheximide (Figure 2B). However, when treated with either $100 \mu \mathrm{M}$ or $250 \mu \mathrm{M}$ CK-666 and cycloheximide, cells exhibit a dose-dependent decrease in ciliary length after 2 hours of regeneration (Figure 2B).

To confirm the results of chemical perturbation, we used the arpc4 mutant strain to genetically test the role of the Arp2/3 complex in incorporation of existing protein. When arpc4 mutant cells were deciliated and treated with cycloheximide, they display minimal growth (Figure 2C). In fact, throughout a five-hour period, only $6 \%$ of cells were able to form cilia at all (Figure 2C). This suggests that the Arp2/3 complex and the actin networks nucleated by the complex are indispensable for the incorporation of existing protein and membrane during ciliary assembly. This can be further dissected because we know in the case of cells treated with cycloheximide and CK-666, the protein pool is available but is still not incorporated, suggesting a problem with membrane incorporation or delivery as opposed to a problem with protein availability.

A

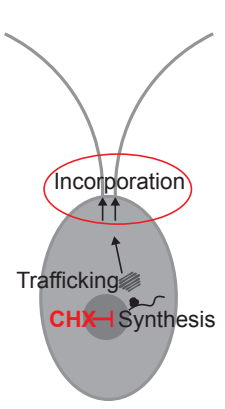

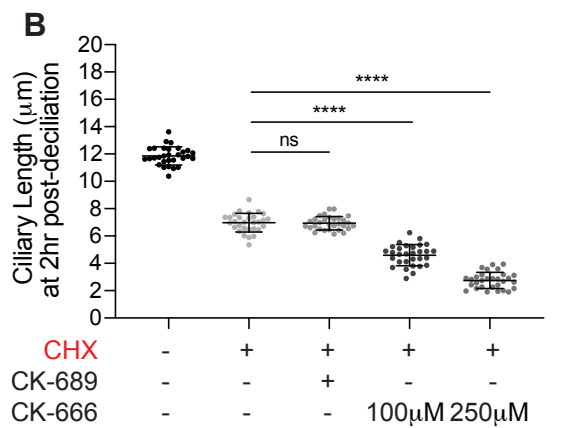

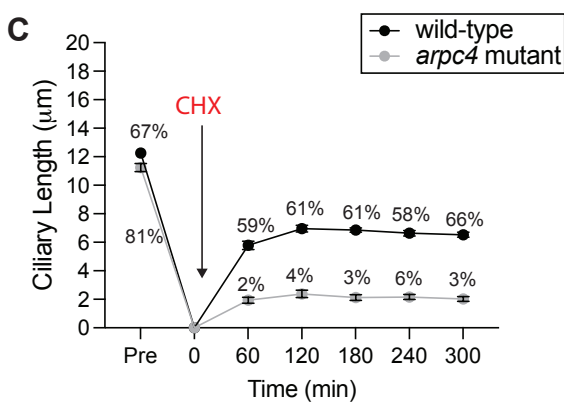

Figure 2. The Arp2/3 complex is required for incorporation of existing protein during ciliary assembly. A) Treating cells with cycloheximide inhibits protein synthesis, which means only incorporation of existing protein into the 
cilia is observed. B) Wild-type cells were deciliated and then treated with a combination of $10 \mu \mathrm{M}$ cycloheximide $(\mathrm{CHX})$ and $\mathrm{CK}-666(100 \mu \mathrm{M}$ or $250 \mu \mathrm{M})$ or CK-689 (the inactive control) at the same concentration during regrowth. The graph shows the length of their cilia after 2 hours of treatment and regrowth. The mean is show with error bars representing standard deviation. $\mathrm{n}=30$ for each treatment group 3 separate experiments. For both the $100 \mu \mathrm{M}$ and $250 \mu \mathrm{M}$ CK-666 treatments with $\mathrm{CHX}, \mathrm{P}<0.0001$. C) Wild-type cells and arpc4 mutants were deciliated and then allowed to regrow in $10 \mu \mathrm{M} \mathrm{CHX}$. The percentages above the lines represent the percent of cells with cilia at the indicated time points. The mean is shown with error bars representing $95 \%$ confidence interval. $n=30$ for each strain and each time point in 3 separate experiments. For every time point besides $0 \mathrm{~min}, \mathrm{P}<0.0001$ for both length and percent ciliation.

\section{Cilia of arpc4 mutant cells resorb faster in the absence of the Golgi:}

Because we see defects in ciliary assembly and maintenance when cells are likely incorporating existing protein, and we know the protein needed for assembly is in excess due to our acute perturbations with CK-666, we next investigated membrane delivery to cilia. This is of particular interest as the Arp2/3 complex is canonically thought to be involved in membrane remodeling functions. Typically, the Golgi is known to be the main source of membrane for cilia (Nachury, Seeley, and Jin 2010; Rohatgi and Snell 2010), and both ciliary membrane, membrane proteins, and even axonemal proteins are transported in or attached to vesicles (Wood and Rosenbaum 2014). In Chlamydomonas, this has been demonstrated by the ciliary shortening of cells treated with Brefeldin A (BFA), a drug that causes Golgi collapse by interfering with ER to Golgi transport (W. Dentler 2013). To determine if the Arp2/3 complex is involved in the trafficking of new protein from the Golgi to cilia, we examined the Golgi following deciliation using transmission electron microscopy (TEM) in arpc4 mutants (Supplemental Figure 3A). The Golgi appeared grossly normal, and in all cases had approximately the same number of cisternae (Supplemental Figure 3A-B) and did not show an abnormal accumulation of post-Golgi membrane as previously reported when perturbing all filamentous actin (Jack et al., 2019).

Alternative pathways for ciliary material have also been found in Chlamydomonas. In previous experiments, surface proteins were biotinylated and then cells were deciliated, meaning the membrane and proteins within cilia were lost. When cilia were allowed to regrow, biotinylated proteins were found to reside within the new cilia suggesting they came from the plasma membrane (W. Dentler 2013). Therefore, we hypothesized that due to its role in membrane remodeling, and particularly endocytosis in other organisms, the Arp $2 / 3$ complex may be part of an endocytic pathway that provides membranes and perhaps membrane proteins to cilia (Figure 3A). To test if membrane could be coming from an endosomal or endocytic source other than the Golgi, we treated cells with $36 \mu \mathrm{M}$ BFA to collapse the Golgi so cells would be forced to utilize other sources of ciliary proteins and membranes. Wild-type cells treated with BFA resorb slowly, but arpc4 mutant cells had a faster resorption rate (Figure 3B and D). Further, the number of cells with cilia in the arpc4 mutant cells dramatically decreased with BFA treatment (Figure 3C). Meanwhile, cells treated with known-ciliary resorption-inducing drugs that don't specifically target Golgi traffic, 3-isobutyl-1-methylxanthine (IBMX) (Pasquale and Goodenough 1987) or sodium pyrophosphate (NaPPi) (P. A. Lefebvre et al. 1978) show an increased velocity of resorption in the wild-type cells compared to the arpc4 mutant cells (Supplemental Figure 4). Thus, wild-type cells are more capable of maintaining cilia without membrane supply from the Golgi, suggesting that there must be another source for membrane that is dependent upon the Arp2/3 complex. 


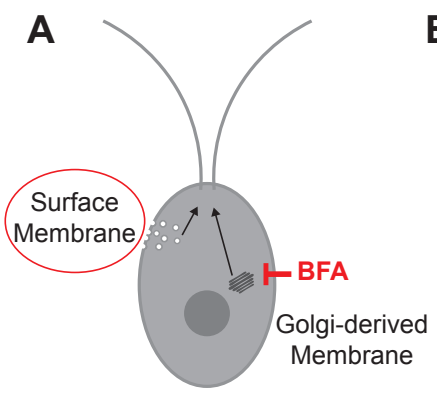$$
\text { B }
$$

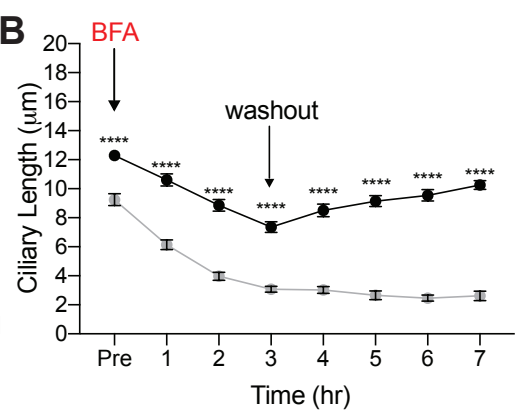

\section{Apical actin dots are dependent on the Arp2/3 complex:} represent endocytic membrane remodeling.

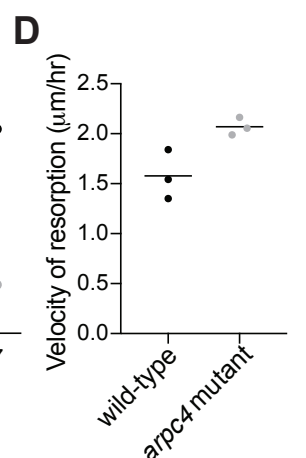

Figure 3. The Arp2/3 complex is required for ciliary maintenance in the absence of intact Golgi. A) Treating cells with BrefeldinA (BFA) causes the Golgi to collapse meaning any membranes and proteins used to maintain the cilia must come from other sources. B) Cells were treated with $36 \mu \mathrm{M}$ BFA for 3 hours at which time the drug was washed out. Wild-type is represented by black, while arpc4 mutants are grey. The mean is shown with error bars representing $95 \%$ confidence interval. Error bars represent $95 \%$ confidence interval of the mean. $n=30$ for each time point and each strain in 3 separate experiments. ${ }^{* * *}$ represents $P<0.0001$. C) Percent ciliation of the cells in $B$. $n=100$. D) Resorption speed for wild-type cells and arpc4 mutant cells as determined by fitting a line to the first 4 time points before washout and determining the slope of the line. Line represents the mean of 3 separate experiments. $N=3$. $P=0.0314$

Since ciliary membrane proteins can come from the Golgi or the plasma membrane and arpc4 mutant cells have a more severe defect in incorporating ciliary proteins from non-Golgi sources, we asked if Arp2/3 complex-mediated actin networks might be responsible for endocytosis from the plasma membrane in Chlamydomonas as it is in other organisms. To determine where in the cell Arp2/3 complex-mediated actin networks might be acting, we looked directly at the effects of loss of Arp2/3 complex function on the actin structures in the cell. Using new protocols for the visualization of actin in Chlamydomonas developed by our lab (Craig et al. 2019), we stained wild-type cells and arpc4 null mutant cells with fluorescent phalloidin. In wildtype cells, apical dots reminiscent of endocytic actin patches in yeast are typically seen near the base of cilia (Figure 4A and $A^{\prime}$ ). We quantified the presence of these dots in the wild-type cells compared to the arpc4 mutant cells (Figure 4B and B'). We found that while about $70 \%$ of wildtype cells contain the dots, only about $5 \%$ of the arpc4 mutant cells had dots (Figure $4 \mathrm{C}$ ), suggesting the Arp2/3 complex is required for the formation of this actin structure. The reliance of this structure on the Arp2/3 complex, led us to further question whether these dots could

A

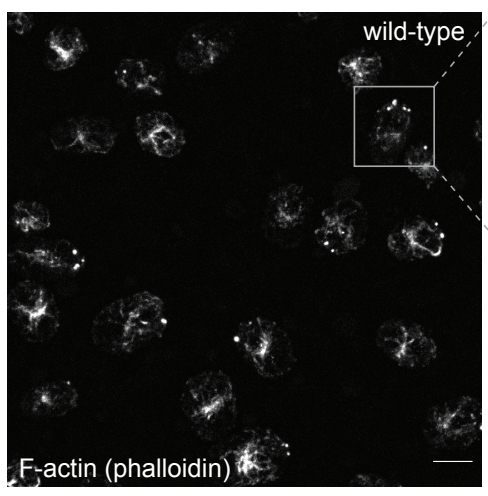

B

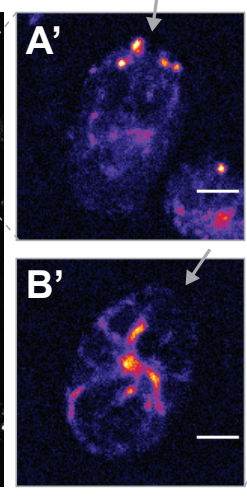

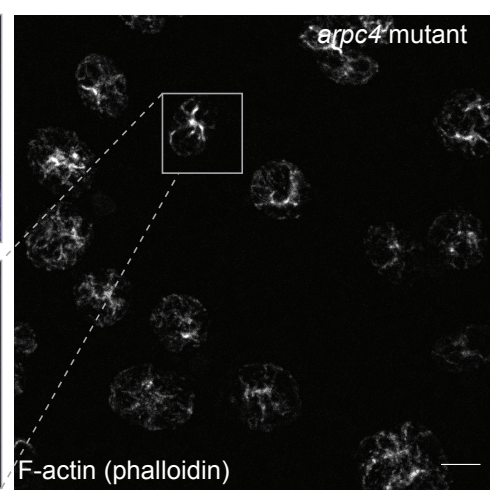

C

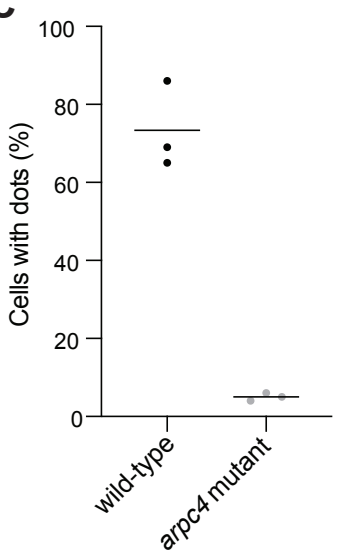


Figure 4. Loss of a functional Arp2/3 complex results in changes in actin distribution. A) Wild-type stained with phalloidin to visualize the actin network. Images were taken as a z-stack using spinning disk confocal imaging and are shown as a maximum intensity projection. Scale bar represents $10 \mu \mathrm{m}$. A' shows the mid-cell actin network of the wildtype cells. The actin distribution is highlighted using the Fire LUT which highlights regions with the brightest intensity in yellow/orange and the areas with less intensity in blue. Grey arrow is pointing to the apex of the cell. Scale bar represents $2 \mu \mathrm{m}$. B) arpc4 mutant cells stained with phalloidin to visualize actin. Cells were imaged and are shown in the same way as A. Scale bar represents $10 \mu \mathrm{m}$. B' shows lack of apical dots and the mid-cell actin staining of arpc4 mutant cells. Image is shown in the same way as A'. Grey arrow is pointing to the apex of the cell. Scale bar represents $2 \mu \mathrm{m}$. C) Percentage of cells with apical dots as shown in A'. Percentages taken from 3 separate experiments where $\mathrm{n}=100$. Line represents the mean. $\mathrm{P}<0.0001$.

\section{Endocytosis in Chlamydomonas is clathrin-dependent:}

The Arp2/3 complex is conventionally thought to be involved in endocytosis in cell-walled yeast to overcome turgor pressure (Aghamohammadzadeh and Ayscough 2009; Basu, Munteanu, and Chang 2014; Carlsson and Bayly 2014). Chlamydomonas cells also have a cell wall and since the apical actin dots resemble these endocytic pits (Goode, Eskin, and Wendland 2015; Adams and Pringle 1984; Ayscough et al. 1997), we hypothesized that Arp2/3 complex and actin-dependent endocytosis might be occurring in Chlamydomonas even though this process has not yet been directly demonstrated in this organism. We compared the endocytosis-related proteins found in mammals and plants to those in Chlamydomonas (Figure 5A). We found that Chlamydomonas was lacking much of the important machinery for almost all typical endocytosis processes, including caveolin for caveolin-mediated endocytosis, flotillin for flotillin-dependent endocytosis, and endophilin for endophilin-dependent endocytosis (Figure 5A). However, most of the canonical clathrin-related endocytosis machinery could be found in Chlamydomonas, suggesting that clathrin-mediated endocytosis is conserved to a higher extent than other endocytic mechanisms.

We further probed the likelihood of clathrin-mediated endocytosis occurring in Chlamydomonas by treating cells with PitStop2 which inhibits the interaction of adaptor proteins with clathrin, halting clathrin-mediated endocytosis itself. The ability of PitStop2-treated cells to internalize membrane was tested using the membrane dye FM 4-46FX, which is usually quickly endocytosed into cells showing bright foci where dye is enriched in endocytosed compartments (Figure 5B). We found that cells treated with $30 \mu \mathrm{M}$ PitStop2 have significantly decreased membrane internalization, meaning clathrin is required for the internalization of this membrane dye (Figure 5C), which further supports the idea that clathrin-mediated endocytosis is occurring in Chlamydomonas.

Finally, we tested whether the endocytosis is Arp2/3 complex-dependent by using this membrane internalization assay on arpc 4 mutant cells compared to wild-type cells. We found that cells lacking a functional Arp2/3 complex have decreased total cell fluorescence (Figure 5D-E) suggesting the clathrin-mediated endocytosis in Chlamydomonas is Arp2/3 complexdependent. 


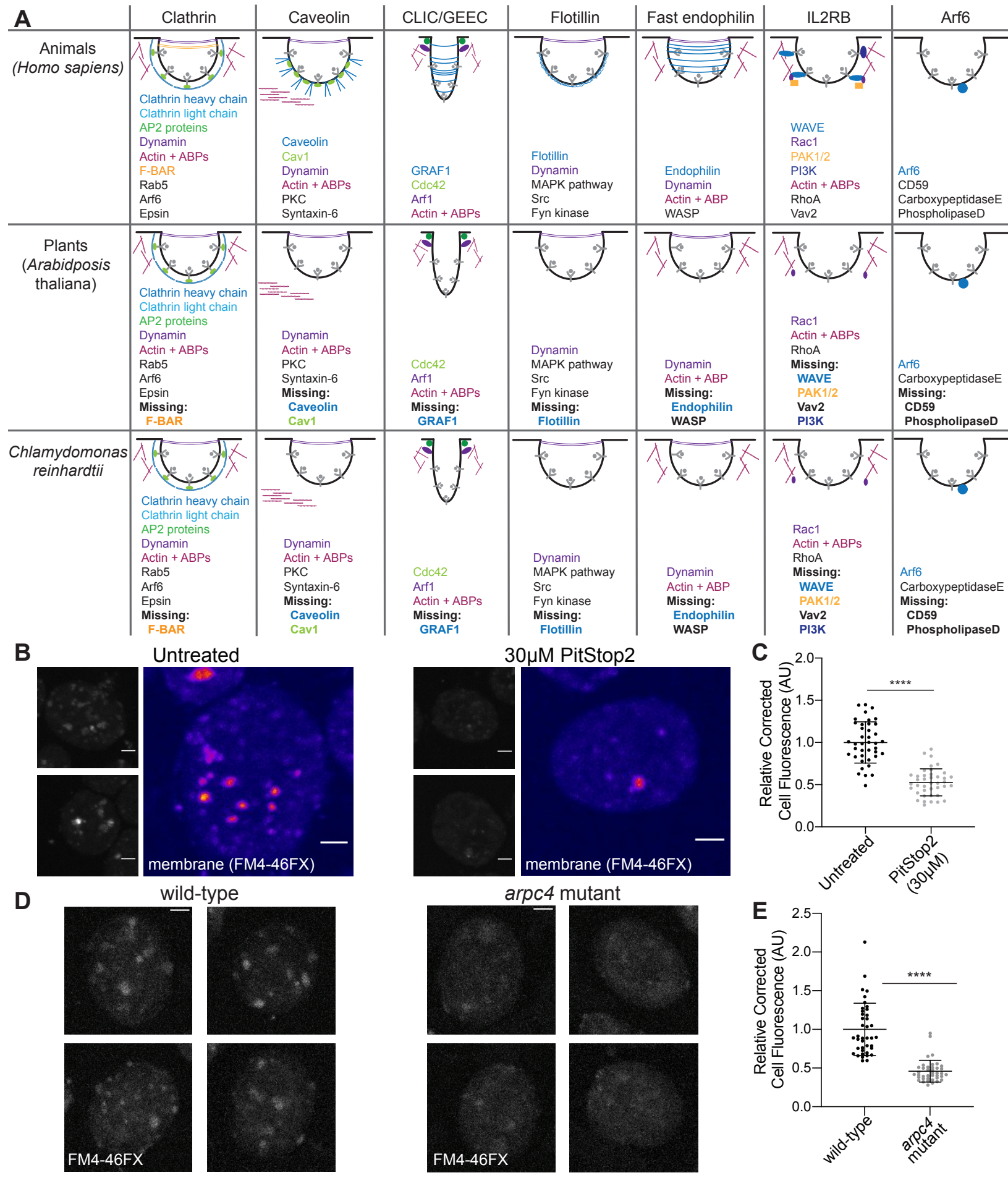

Figure 5. Arp2/3 complex-dependent clathrin-mediated endocytosis is conserved in Chlamydomonas. A) Gene

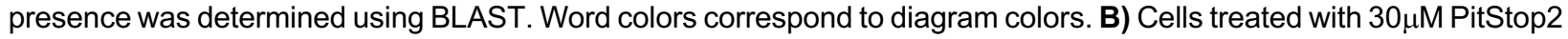
were incubated with FM4-46FX and imaged on a spinning disk confocal. Max intensity projections of Z-stacks are shown. Scale bars are $2 \mu \mathrm{m}$. C) The background corrected fluorescence for each sample. The mean is shown with error bars showing standard deviation. $\mathrm{n}=40$ in 3 separate experiments. $\mathrm{P}<0.0001$. D) Wild-type and arpc4 mutant cells treated with FM4-46FX and imaged on a spinning disk confocal. Max intensity projections of Z-stacks are shown. Scale bars are $2 \mu \mathrm{m}$. E) The background corrected fluorescence for each sample. The mean is shown with error bars representing standard deviation. $n=30$ in 3 separate experiments. $P<0.0001$. 
The Arp2/3 complex is required for the internalization and relocalization of a membrane protein from the periphery of the cell to cilia:

Upon finding that there is likely Arp2/3 complex-dependent clathrin-mediated endocytosis in Chlamydomonas, we next asked if this endocytosis could be responsible for the relocalization and internalization of a known ciliary protein. SAG1 is a membrane protein that relocalizes to cilia and is important for helping cells stick together or agglutinate in mating Chlamydomonas cells (Belzile et al. 2013). When cells are induced for mating with dibutyrylcAMP (db-cAMP), SAG1 must relocalize from the cell periphery to cilia, where it facilitates ciliary adhesion between mating cells. This relocalizaiton of SAG1 is thought to occur through internalization of the protein followed by internal trafficking on microtubules to the base of cilia (Belzile et al. 2013).

We examined whether actin and the Arp2/3 complex were required for the transport of HA-tagged SAG1 to the apex of the cell and cilia for agglutination during mating (Figure 6A). Using immunofluorescence, we observed cells treated with either $10 \mu \mathrm{M}$ LatB to depolymerize IDA5 or $250 \mu \mathrm{M}$ CK-666 to perturb the Arp2/3 complex. Before induction, SAG1-HA localized to the periphery of the cell (Figure 6B, top). 30 minutes after induction with db-cAMP, SAG1-HA relocalized to the apex of the cell and to cilia in untreated cells (Figure 6B, left). In both LatB and CK-666 treated cells, this apical enrichment was greatly decreased (Figure 6B, middle and right). To quantify this, line scans were drawn through the cell from the apex to the basal region (Figure 6C-D). The percentage of cells with apical enrichment was calculated, and it was found that untreated cells had a higher percent of apical enrichment when compared with LatB or CK-666 treated cells (Figure 6E). Thus, cells with perturbed Arp2/3 complex-mediated filamentous actin show decreased efficiency of SAG1-HA relocalization.

We next asked if this decrease in relocalization in cells with actin and Arp2/3 complex inhibition could be due to a decrease in the internalization of SAG1-HA through a process that seems to require endocytosis. In order to investigate this, we used a method first described by Belzile et al. 2013, where cells were induced and treated with a low percentage $(0.01 \%)$ of trypsin which will hydrolyze exterior proteins but cannot enter the cell. This assay allows us to determine the efficiency of internalization of SAG1-HA. In untreated cells, we see an increase in SAG1-HA protein levels following induction because SAG1-HA is internalized and becomes protected from trypsin (Figure 6F). In cells treated with either $10 \mu \mathrm{M}$ LatB or $250 \mu \mathrm{M}$ CK-666 we see a decrease in this trypsin protection as shown in the western blot (Figure 6F). This was further quantified by subtracting the amount of protein before induction from the amount of protein present after induction. This gives a value representing the amount of SAG1-HA protected from trypsin due to induction in these cells (Figure 6G). The decrease in SAG1-HA following induction in cells with decreased filamentous actin and Arp2/3 complex function indicates a role for Arp2/3 complex-mediated actin networks in internalization of this ciliary protein. 

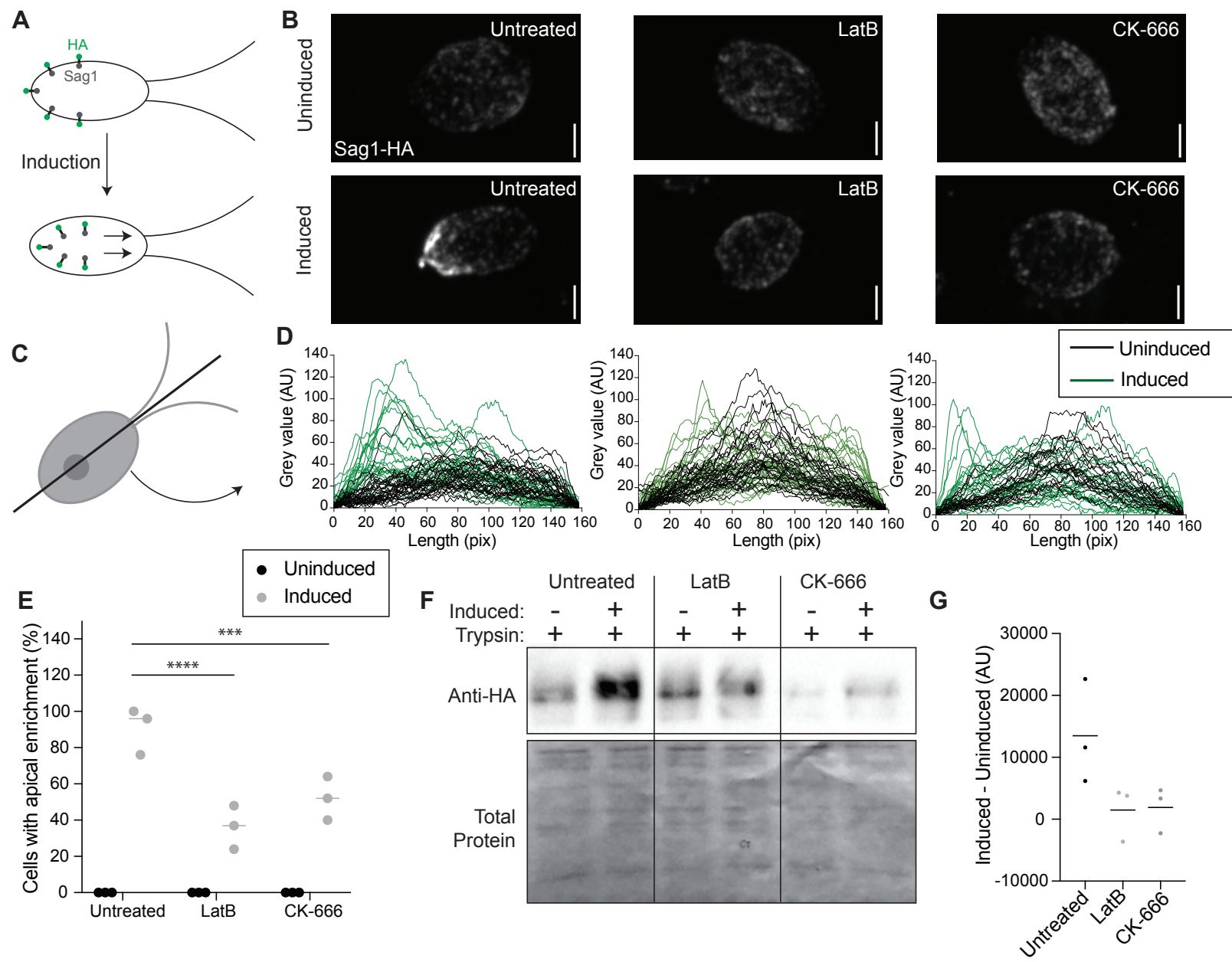

Figure 6. The Arp2/3 complex is required for the relocalization and internalization of the ciliary protein SAG1 for mating. A) When mating is induced SAG1-HA is internalized and relocalized to the apex of the cells and cilia for agglutination. B) Maximum intensity projections of z-stacks taken using spinning disk confocal microscopy of SAG1HA. Left is untreated, middle is treated with $10 \mu \mathrm{M}$ LatB, and right is treated with $250 \mu \mathrm{M}$ CK-666. Top row of images are uninduced and bottom row of images are induced with db-cAMP. Scale bar represents $2 \mu \mathrm{m}$. C) Diagram representing line scans taken through the cells in z-stack sum images. D) Line scans from the apex of the cell to the basal region of the cell in untreated cells (left), LatB treated cells (middle), and CK-666 (right). Black lines represent uninduced samples, green lines represent induced samples. E) Percentage of cells with apical enrichment based on E for uninduced (black) and induced (grey) cells for each treatment group. The mean is shown with error bars representing standard deviation. $\mathrm{n}=30$ for 3 separate experiments for each treatment. F) Western blot showing amount of SAG1-HA in uninduced and induced cells in each treatment group all treated with $0.01 \%$ trypsin. G) Intensity of the bands in $\mathrm{H}$ were normalized to the total protein as determined by amido black staining and quantified in ImageJ was used to subtract uninduced from induced to give a representation of the amount of SAG1-HA internalized with induction. Line represents mean of 3 separate experiments.

\section{Actin dots increase in an Arp2/3 complex and clathrin-dependent manner following deciliation:}

Having established that the Arp2/3 complex is required for ciliary assembly, the endocytosis of a known ciliary protein, and membrane dye internalization, we wondered if these functions could be connected given that arpc4 mutant cells have defects in maintaining cilia from non-Golgi sources (Figure 3). Therefore, we returned to the clathrin and Arp2/3 complexdependent actin dots seen in wild-type cells that are reminiscent of endocytic pits in yeast. Because ciliary membrane and proteins can come from the plasma membrane (Dentler, 2013), 
we suspected there would be an increase in these actin dots immediately following deciliation. We used phalloidin to visualize the actin cytoskeleton of wild-type cells before and immediately following deciliation, as well as 10 minutes later (Figure 7A). We saw an increase in both the percentage of cells with dots and the number of dots per cell immediately following deciliation that then returned to normal by 10 minutes (Figure 7A-B). Importantly, this is consistent with the results shown in Figure 1D-E and confirms that the defect seen in ciliary assembly is due to an event occurring very early in ciliary assembly, even within the first few minutes after deciliation.

We wondered if this increase in dots was really due to clathrin-mediated endocytosis, so we treated cells with PitStop2 and looked for this same increase in dots. This treatment almost fully blocked the appearance of dots following deciliation and completely eliminated the presence of cells with 3 or more dots (Figure 7C-D), suggesting a clathrin-dependent mechanism is related to these dots. Further, we wanted to know if this increase in actin dots relied on the Arp2/3 complex, so we looked for dots following deciliation in arpc4 mutant cells (Figure 7E). We found that in the arpc4 mutants dots were never observed, before or after deciliation (Figure 7E), suggesting these dots are dependent on not just clathrin function, but 400

A

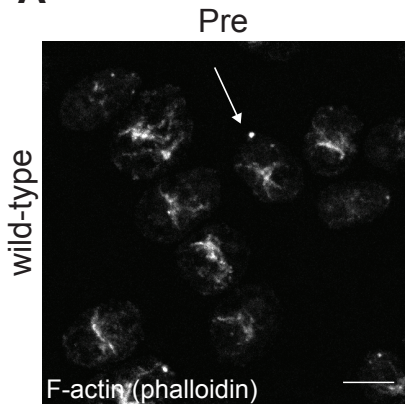

C

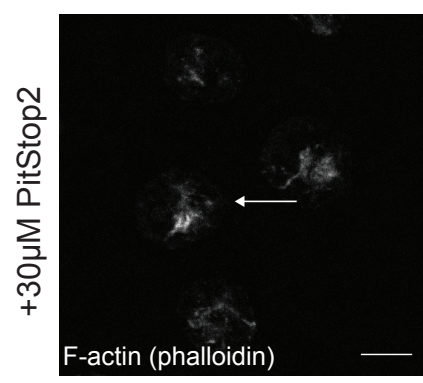

E

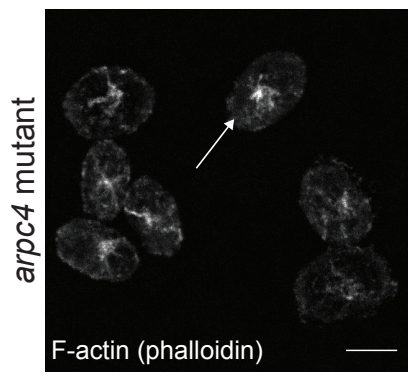

0 minutes following deciliation
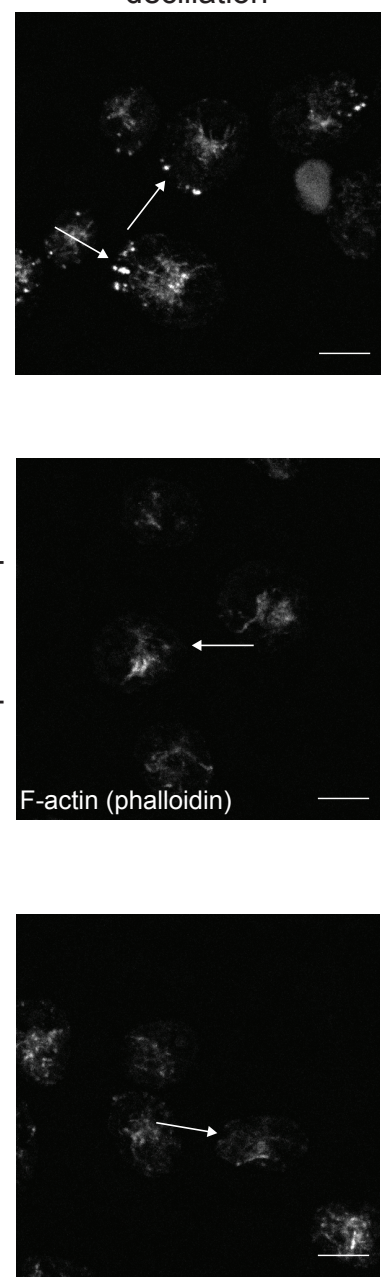

10 minutes following deciliation
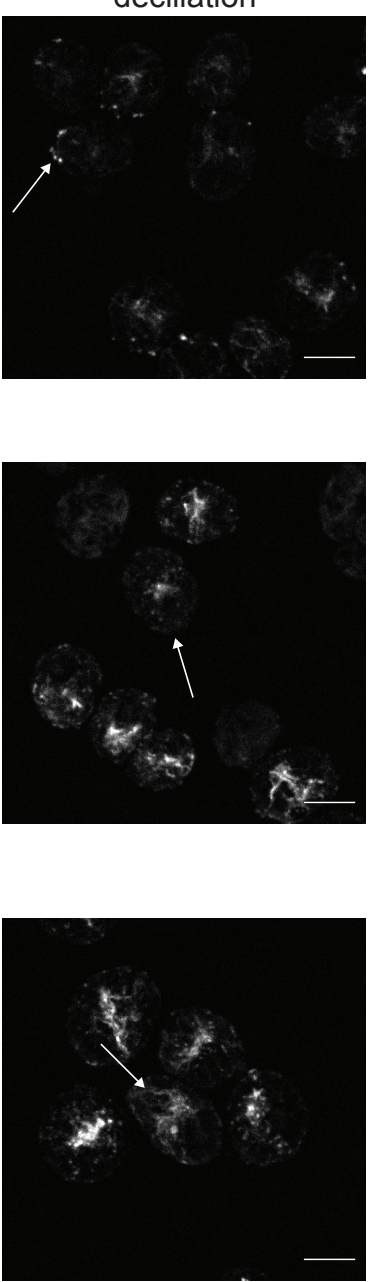

B
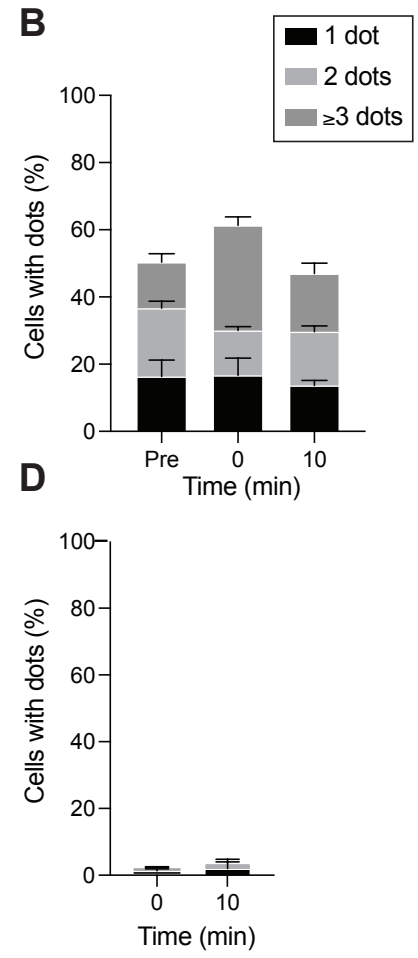

401 
Figure 7. Actin dots are clathrin and Arp2/3 complex-dependent. A) Wild-type cells stained with phalloidin to visualize the actin network before deciliation, immediately following deciliation, and 10 minutes following deciliation. Images were taken as a z-stack using spinning disk confocal imaging and are shown as a maximum intensity projection. Scale bar represents $5 \mu \mathrm{m}$. Arrows point to the apex of the cell. B) The percentage of wild-type cells with 1 dot, 2 dots, or 3 dots in Pre, 0 minute, and 10-minute samples. The bars represent the mean with error bars showing standard

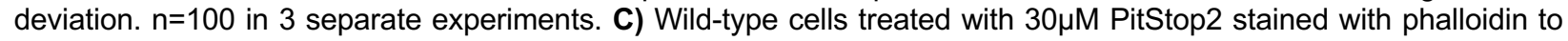
visualize the actin immediately following deciliation and 10 minutes following deciliation. Images were taken as a zstack using spinning disk confocal imaging and are shown as a maximum intensity projection. Scale bar represents $5 \mu \mathrm{m}$. Arrows point to the apex of the cell. D) The percentage of wild-type cells treated with PitStop2 with 1 dot, 2 dot, or 3 dots in 0 minute and 10-minute samples. $\mathrm{n}=100$ in 3 separate experiments. E) arpc4 mutant cells stained with phalloidin before deciliation, immediately following deciliation, and 10 minutes following deciliation. Arrows point to the apex of the cells.

\section{Clathrin localization post deciliation is altered in the absence of the Arp2/3 complex:}

To directly probe the involvement of clathrin and clathrin-mediated endocytosis in ciliary assembly, we stained cells with an antibody for clathrin light chain (Figure 8). Wild-type cells showed an increase in clathrin signal following deciliation, perhaps due to an increase in expression (Figure 8). In the arpc4 mutant cells following deciliation, we see an accumulation of clathrin in the area of the cell opposite from the cilia and the nucleus (Figure 8, Supplemental Figure 5). This accumulation is also observed following deciliation in wild-type or arpc4 mutant cells treated with PitStop2 to block clathrin-mediated endocytosis (Figure 8), suggesting that the loss of Arp2/3 complex function and the loss of clathrin-mediated endocytosis may be linked.
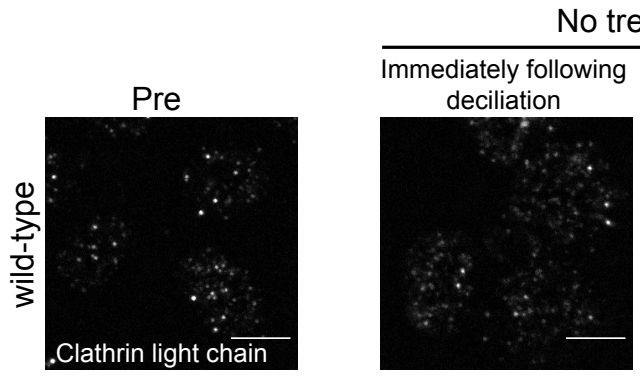

No treatment
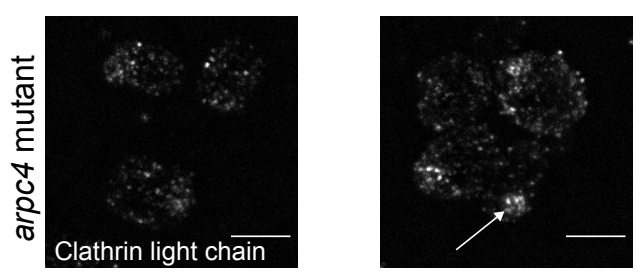
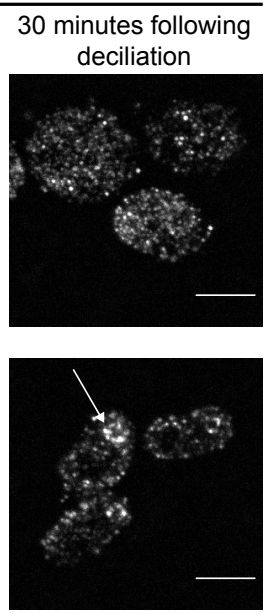

PitStop2

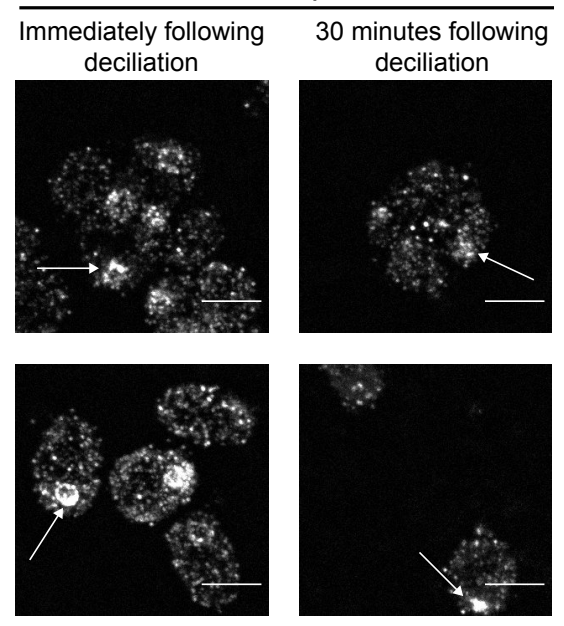

Figure 8. Clathrin is mislocalized in cells treated with PitStop2 or lacking the Arp2/3 complex. Untreated or $30 \mu \mathrm{M}$ PitStop2 treated wild-type and arpc4 mutant cells stained with clathrin light chain antibody to visualize clathrin localization before deciliation, immediately following deciliation, and 30 minutes following deciliation. Images were taken as a z-stack using spinning disk confocal imaging and are shown as a maximum intensity projection. Arrows point to mislocalized clathrin light chain. Scale bar represents $5 \mu \mathrm{m}$.

\section{Ciliary membrane proteins follow different paths from the plasma membrane to the cilia:}

Finally, to specifically determine if ciliary membrane and therefore membrane proteins were coming from a pool in the plasma membrane we did an experiment first described in (W. Dentler 2013). Surface proteins were biotinylated, then cells were deciliated. After the cilia regrew, they were isolated and probed for biotinylated protein (Figure 9A). While some proteins returned in both wild-type and arpc4 mutant cells, some appeared to a lesser degree in arpc4 
mutant cells compared to wild-type cells (Figure 9B-E, black arrow and black bars) and some returned to a higher degree in arpc4 mutant cells (Figure 9B-E, grey arrow and grey bars). Other biotinylated proteins found in wild-type cilia were not found in the arpc4 mutant cilia before or after deciliation, so there is a mechanism for delivery of proteins to the cilia from the plasma membrane that Arp2/3 is absolutely essential for (Figure 9B-C). This suggests there are multiple paths to the ciliary membrane, some of which are Arp2/3 complex-independent and some that are Arp2/3 complex-dependent. This may represent lateral diffusion and endocytosis 448 respectively.

449
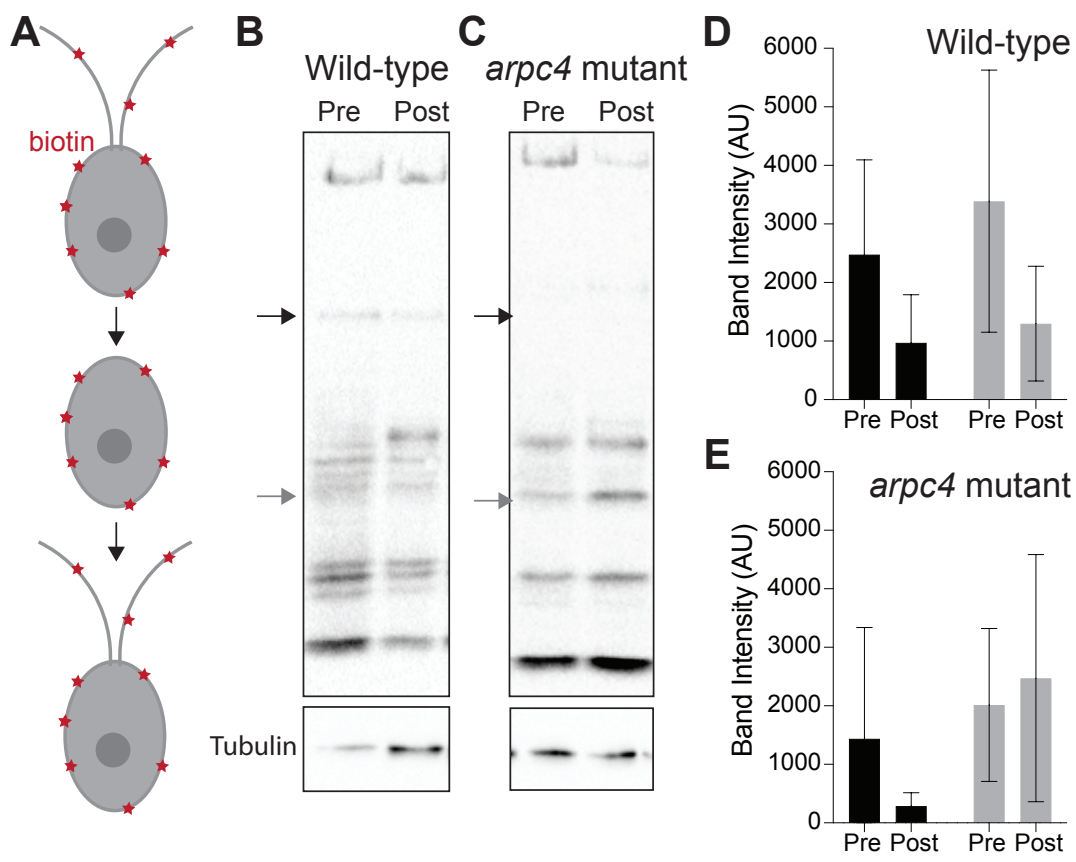

Figure 9. Ciliary membrane proteins have multiple paths from the plasma membrane. A) Cells were biotinylated, deciliated, and then allowed to regrow before cilia were isolated and probed for biotinylated protein. B) Wild-type ciliary isolate was then investigated by western blot and probed using streptavidin and tubulin as a loading control. C) arpc4 mutant cells ciliary isolate was investigated by western blot and probed using streptavidin. Black arrow shows ciliary protein present to a higher degree in wild-type cells than the arpc4 mutant cells. Grey arrows show ciliary protein that is present to a higher degree in arpc4 mutant cells than in wild-type cells. D) Bands represented by black and grey arrows are quantified for the wild-type cells. Data acquired from 3 separate experiments. E) Bands represented by black and grey arrows are quantified for the arpc4 mutant cells. Data represented as the mean from 3 separate experiments. Error bars represent standard deviation.

\section{DISCUSSION}

In this study, we investigate the Arp2/3 complex of Chlamydomonas reinhardtii that functions to maintain and assemble cilia. This complex potentially lacks the ARPC5 subunit, although it is possible that a highly divergent ARPC5 exists. In yeast, deletion of any of the genes encoding Arp2/3 complex members causes severe defects and even lethality, but these defects differ in severity depending on the complex members deleted, suggesting that complex members have varying degrees of importance in Arp2/3 complex function (Winter, Choe, and Li 1999). The role of ARPC5 in actin nucleation is being investigated, but several groups have found it unnecessary for overall function of the complex (Gournier et al. 2001; von Loeffelholz et al. 2020). Furthermore, our data shows that knocking out function of the ARPC5-less Chlamydomonas Arp2/3 complex genetically or chemically results in phenotypes suggesting 
that the active complex functions in ciliary assembly and maintenance. Because the Arp2/3 complex has known functions in membrane dynamics, this led us to pursue models of Arp $2 / 3$ complex-dependent membrane trafficking to cilia.

Previously, three models for the trafficking of membrane proteins to cilia have been proposed (Nachury, Seeley, and Jin 2010). The first is that Golgi vesicles containing ciliary proteins are docked at the base of the cilium and fuse with the ciliary membrane inside the cilium itself. Proteins, both membrane and axonemal, have been found to be transferred from the Golgi to the cilia on or in cytoplasmic vesicles (Wood and Rosenbaum 2014). Second, Golgi vesicles containing ciliary proteins are docked near the base of the cilium still within the diffusion barrier (Papermaster, Schneider, and Besharse 1985; Nachury et al. 2007; Zuo, Guo, and Lipschutz 2009). In Chlamydomonas, this was first described for mastigoneme proteins, which were found to be transferred from the Golgi and then exocytosed for use on the exterior of the cell (Bouck 1971). Finally, Golgi vesicles containing proteins fuse with the plasma membrane and membrane proteins are then somehow moved across this barrier within the plasma membrane, perhaps through lateral diffusion. Evidence for this path was shown using Hedgehog signaling protein Smo, which was found to relocalize in a dynamin-independent manner from the plasma membrane to the cilia immediately after stimulation in pulse labeling studies (Milenkovic, Scott, and Rohatgi 2009).

Our data support a fourth model for the recruitment of a reservoir of membrane proteins to the cilium that is likely occurring in concert with the other models. We find the Arp $2 / 3$ complex is required for ciliary assembly, clathrin-mediated endocytosis, redistribution of ciliary proteins from the plasma membrane, and all of these activities happen immediately post-deciliation (Figure 10A). We hypothesize that ciliary membrane proteins and membrane targeted to the plasma membrane of the cell outside the diffusion barrier can be endocytosed and trafficked to cilia, either within or outside of the diffusion barrier in an actin and Arp2/3 complex-dependent manner.

Although our data does not eliminate the possibility of Arp2/3 complex function in supply of ciliary membrane and protein stored in other endosomal compartments, ciliary localization of proteins initially labeled on the cell surface with biotin (Figure 9) suggests that some ciliary membrane proteins incorporated during assembly are coming directly from the plasma membrane itself. An endocytic mechanism of trafficking in intracellular ciliogenesis has been investigated previously in mammalian RPE1 cells. The ciliary pocket found at the base of primary and motile cilia formed intracellularly has been found to be an endocytically active region (Molla-Herman et al. 2010) but clathrin mediated endocytosis was not required for ciliogenesis in those cells. The Bardet Biedl Syndrome complex (BBsome), which is involved in regulation of ciliary membrane protein composition, has been shown to interact with clathrin directly at the ciliary pocket to facilitate membrane sorting in trypanosomes (Langousis et al. 2016). Further, some BBsome complex members resemble coat proteins such as clathrin (Jin et al. 2010) suggesting a direct role for the this cilium regulatory complex in membrane budding functions. Even in Chlamydomonas, clathrin heavy chain has been found to localize at the base of cilia (Kaplan et al. 2012). While the mechanism was unknown, it has been shown that plasma membrane surface-exposed proteins are relocalized to cilia during ciliary regeneration (W. Dentler 2013), a result we were able to recapitulate and demonstrate depends in $_{2}$ part ${ }_{2}$ upon the Arp2/3 complex.

Altogether, this leads us to hypothesize that the role of the Arp2/3 complex in ciliary assembly is through endocytic recruitment from a ciliary protein reservoir in the plasma membrane before newly synthesized protein and Golgi-derived membrane are capable of supplying additional materials (Figure 10B). While this model provides a possible route that some ciliary proteins and membranes take to the cilia, we believe this is one of several paths that can be taken to the cilia. Trafficking to cilia is likely cargo- and time-dependent, and which 
bioRxiv preprint doi: https://doi.org/10.1101/2020.11.24.396002; this version posted April 1, 2021. The copyright holder for this preprint (which was not certified by peer review) is the author/funder, who has granted bioRxiv a license to display the preprint in perpetuity. It is made available under aCC-BY 4.0 International license.

525

526

527

path proteins take may tell us the order and speed in which they populate the cilium for subsequent function.

A

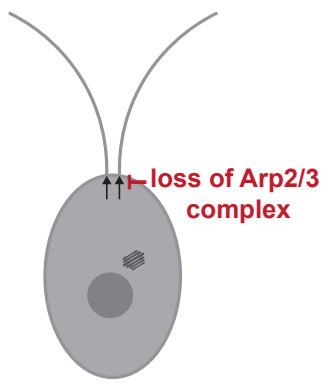

Early ciliary assembly is Arp2/3 complexdependent

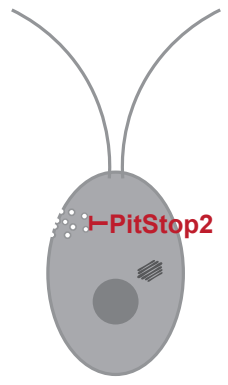

Arp2/3 complexdependent clathrinmediated endocytosis occurs in Chlamydomonas

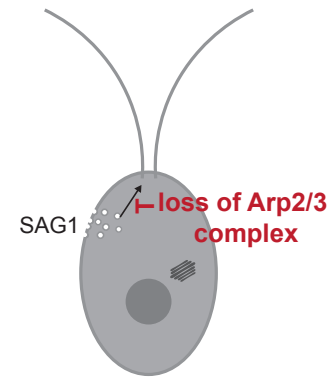

Relocalization of a plasma membrane protein to cilia is Arp2/3 complex-dependent

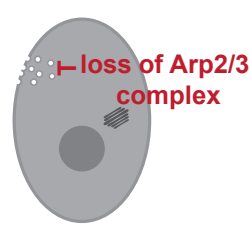

Arp2/3 complex and clathrin-dependent endocytic event occurs during early ciliary assembly

\section{B}

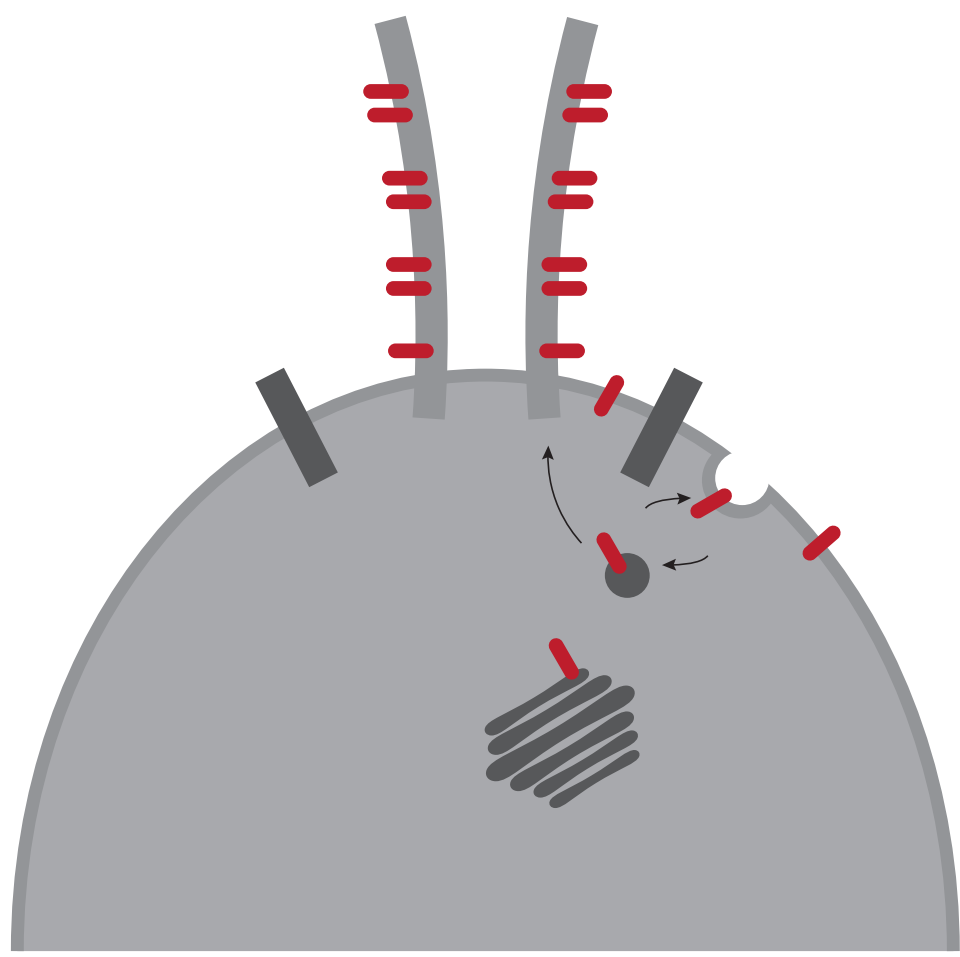

Figure 10. The Arp2/3 complex is required for membrane and protein delivery via a Golgi-independent, endocytosis-like process. A) Arp2/3-mediated actin networks are required for ciliary assembly in Chlamydomonas particularly during the initial stages. These actin networks are also required for clathrin-mediated endocytosis, and for the endocytosis-like relocalization of a ciliary protein from the plasma membrane to the cilia. Finally, a large endocytic event occurs immediately following deciliation that is Arp2/3 complex-mediated and dependent on clathrin-mediated endocytosis. B) Proposed model of membrane protein and membrane transport from the plasma membrane to the cilia through endocytosis. 


\section{METHODS}

\section{Strains:}

The wild-type Chlamydomonas strain (CC-5325) and the arpc4 mutant (LMJ.RY0402.232713) from the Chlamydomonas resource center. The nap1 mutant was a gift from Fred Cross, Masayuki Onishi, and John Pringle. The SAG1-HA strain was a gift from William Snell. Cells were grown and maintained on 1.5\% Tris-Acetate Phosphate Agar (TAP) plates (Chlamydomonas resource center) under constant blue (450-475 nm) and red light (625-660 $\mathrm{nm}$ ). For experiments, cells were grown in liquid TAP media (Chlamydomonas resource center) overnight under constant red and blue light with agitation from a rotator. To induce gametes for mating for the SAG1-HA experiments, cells were grown in liquid M-N media (Chlamydomonas resource center) overnight with constant red and blue light and agitation.

\section{Ciliary studies:}

553 For steady state experiments, cells were treated with specified drugs [either 100 $\mu \mathrm{M} \mathrm{CK}-666$, 250 $\mu$ M CK-666 (Sigma, Burlington, MA), 250 $\mu$ M CK-689 (Sigma, Burlington, MA), 10 $\mu$ M LatB (Sigma, Burlington, MA), 10 $\mathrm{M} \mathrm{CHX} \mathrm{(Sigma,} \mathrm{Burlington,} \mathrm{MA),} \mathrm{or} \mathrm{36 \mu M} \mathrm{BFA} \mathrm{(Sigma,} \mathrm{Burlington,}$ $\mathrm{MA})]$ and incubated with agitation for the allotted times. Following any incubation (as well as a pre sample), cells were diluted in an equal volume of $2 \%$ glutaraldehyde and incubated at $4^{\circ}$ Celsius until they sediment. Following sedimentation, cells were imaged using a Zeiss DIC scope with a $40 \mathrm{X}$ objective. Cilia were then measured using the segmented line function in ImageJ.

For regeneration experiments, a pre sample was taken by adding cells to an equal volume of $2 \%$ glutaraldehyde. Then cells were deciliated with $115 \mu \mathrm{L}$ of $0.5 \mathrm{~N}$ acetic acid for 45 seconds. After this short incubation, the $\mathrm{pH}$ was returned to normal by adding $120 \mu \mathrm{L}$ of $0.5 \mathrm{~N} \mathrm{KOH}$. A $0-$ minute sample was again taken by adding cells to an equal volume of $2 \%$ glutaraldehyde. Then cells were incubated with agitation and allowed to regrow cilia for the allotted time period with samples taken at the indicated time points by adding cells to an equal volume of $2 \%$ glutaraldehyde. Cells in glutaraldehyde were allowed to incubate at $4^{\circ}$ Celsius until sedimentation. Then, cells were imaged using the same Zeiss DIC scope with a 40X objective. Cilia were then measured using the segmented line function in ImageJ.

\section{Phalloidin staining and quantification:}

Procedure adapted from (Craig et al. 2019). Cells were mounted onto poly-lysine coverslips and fixed with fresh $4 \%$ paraformaldehyde in 1X HEPES. Coverslips with cells were then permeabilized with acetone and allowed to dry. Cells were rehydrated with PBS, stained with atto-phalloidin-488 (Sigma, Burlington, MA), and finally washed with PBS and allowed to dry before mounting with Fluoromount-G (Craig et al. 2019). Cells were imaged using the Nikon Spinning Disk Confocal. Z-stacks were obtained, and in ImageJ, maximum intensity projections were created for viewing.

\section{Electron microscopy:}

Cells ( $1 \mathrm{~mL}$ of each strain) were deciliated via pH shock by adding $115 \mu \mathrm{L}$ of $0.5 \mathrm{~N}$ acetic acid for 45 seconds followed by $120 \mu \mathrm{L}$ of $0.5 \mathrm{~N} \mathrm{KOH}$ to bring cells back to neutral $\mathrm{pH}$. Cells were allowed to regrow cilia for 30 minutes. A pre sample and a 30 -minute post-deciliation sample were fixed in an equal volume of $2 \%$ glutaraldehyde for 20 minutes at room temperature. Samples were then pelleted using gentle centrifugation for 10 minutes. The supernatant was removed, and cells were resuspended in $1 \%$ glutaraldehyde, $20 \mathrm{mM}$ sodium cacodylate. Cells were incubated for 1 hour at room temperature and then overnight at $4^{\circ}$ Celsius. This protocol was first reported in (W. L. Dentler and Adams 1992). 


\section{SAG1-HA Immunofluorescence:}

Procedure adapted from (Belzile et al. 2013). SAG1-HA cells were grown overnight in M-N media to induce gametes. These cells were then treated with either $10 \mu \mathrm{M}$ LatB for 1 hour or $250 \mu \mathrm{M}$ CK-666 for 2 hours. Following treatment, mating was induced by adding db-cAMP (ChemCruz, Santa Cruz, CA) to a final concentration of $13.5 \mathrm{mM}$ and incubating for 30 minutes. Cells were adhered to coverslips and fixed with methanol. Cells were then dried and rehydrated with PBS and incubated with 100\% block (5\% BSA, 1\% fish gelatin) for 30 minutes. The $100 \%$ block was replaced with new $100 \%$ block containing $10 \%$ normal goat serum for another 30 minute incubation. The primary antibody (rat anti-HA, Sigma, Burlington, MA) was diluted $1: 1000$ in $20 \%$ block in PBS. Coverslips were incubated at $4^{\circ}$ Celsius in a humidified chamber overnight. The primary antibody was removed and washed away with 3 10-minute PBS washes. The secondary (anti-rat IgG-Alexafluor 488, Invitrogen, Carlsbad, CA) was added and coverslips were incubated at room temperature for 1 hour. This was followed by 3 more 10-minute PBS washes and finally mounting with Fluoromount-G. Cells were imaged using a Nikon widefield microscope. Z-stacks were obtained, and maximum intensity projections were created for visualization and sum slices were created for quantification using ImageJ.

Images were quantified by using line scans from the apex of the cells to the basal region of the cells farthest away from the apex. Line scans were then normalized, and background subtracted before being combined into single graphs. Using the line scans, the intensity of signal at the basal region of the cells was subtracted from the signal at the apical region. Finally, cells with a difference over 30 were considered to be apically enriched and this was quantified as percentage of cells with apical staining.

\section{SAG1-HA western blot:}

Procedure adapted from (Belzile et al. 2013). SAG1-HA cells were grown overnight in M-N media to induce gametes. These cells were then treated with either $10 \mu \mathrm{M}$ LatB for 1 hour or $250 \mu \mathrm{M} \mathrm{CK}-666$ for 2 hours. Following treatment, mating induction was done by adding db-CAMP to a final concentration of $13.5 \mathrm{mM}$ and incubating for 10 minutes. Cells were then treated with $0.01 \%$ trypsin for 5 minutes, pelleted (at $500 \times$ g for 2 minutes), resuspended in lysis buffer ( $5 \%$ glycerol, $1 \%$ NP-40, $1 \mathrm{mM}$ DTT, $1 \mathrm{X}$ protease inhibitors), and then lysed with bead beating. Cell debris was spun down at $14000 x g$ for 15 minutes. An equal amount of protein was loaded to a $10 \%$ SDS-PAGE gel. The resulting gel was transferred to membrane which was then blocked with $5 \%$ milk in PBST. The primary antibody (rabbit anti-HA, Cell Signaling, Danvers, MA) diluted to $1: 1000$ in $1 \%$ BSA, $1 \%$ milk was added and incubated overnight at $4^{\circ}$ Celsius. Primary antibody washed off with 3 10-minute PBST washes. Secondary antibody (anti rabbit IgG, Invitrogen, Carlsbad, CA) was diluted to $1: 5000$ in 1\% milk. 1\% BSA was added, and the blot was incubated for 1 hour. Membrane was probed with West Pico Chemiluminescent Pico Substrate (Invitrogen, Carlsbad, CA). Total protein was determined with amido black staining. Band intensity was measured in ImageJ and normalized to total protein

\section{Membrane stain:}

FM 4-64FX membrane stain (Thermo, Waltham, MA) was diluted to a stock concentration of $200 \mu \mathrm{g} / \mathrm{mL}$. Cells were adhered to poly-lysine coverslips. After a 5-minute incubation, cells were tilted off and $5 \mu \mathrm{g} / \mathrm{mL}$ of ice-cold stain in Hank's Buffered Salt Solution (HBSS) without magnesium or calcium was added for 1 minute. The stain was tilted off and cells were fixed with ice cold $4 \%$ paraformaldehyde in HBSS without magnesium or calcium for 15 minutes. Coverslips were then rinsed 3 times for 10 minutes each in ice cold HBSS without magnesium or calcium. Finally, cells were mounted with Fluoromount-G and imaged using the Nikon 
640

641

642

643

644

645

646

647

648

649

650

651

652

653

654

655

656

657

658

659

660

661

662

663

664

665

666

667

668

669

670

671

672

673

674

675

676

677

678

679

680

681

682

683

684

685

686

687

688

689

Spinning Disk Confocal. Z-stacks were taken and combined into sum projections using ImageJ. The background corrected total cell fluorescence was then calculated by taking the integrated density and subtracting the sum of the area and the mean background intensity.

\section{Clathrin light chain immunofluorescence:}

Cells were grown overnight in TAP media. Cells were deciliated using low pH shock. Cells are then adhered to coverslips and fixed with 4\% PFA in 1X HEPES. Cells were then dried and rehydrated with PBS and incubated with 100\% block (5\% BSA, 1\% fish gelatin) for 1 hour. The primary antibody (goat anti-clathrin light chain, Abcam, Cambridge, UK or rabbit anti-acetylated tubulin, Cell Signaling, Danvers, MA) was diluted 1:1000 in 20\% block in PBS. Coverslips were incubated at $4^{\circ}$ Celsius in a humidified chamber overnight. The primary antibody was removed and washed away with 3 10-minute PBS washes. The secondary (donkey anti-goat IgGAlexafluor 488, Invitrogen, Carlsbad, CA or goat anti-rabbit IgG-Alexafluor 568, Invitrogen, Carlsbad, CA) was added and coverslips were incubated at room temperature for 1 hour. For cells stained with DAPI, DAPI (Biotium, Fremont, CA) was added for the last 10 minutes of secondary antibody incubation. This was followed by 3 more 10-minute PBS washes and finally mounting with Fluoromount-G. Cells were imaged using a Nikon widefield microscope. Z-stacks were obtained, and maximum intensity projections were created for visualization and sum slices were created for quantification using ImageJ.

\section{Biotin ciliary isolation:}

Procedure adapted from (W. Dentler 2013). 100mL of cells were grown in TAP for each condition until they reached an $\mathrm{OD}_{730}$ of 1.6 or above. Cells were then spun down and resuspended in M1 media and allowed to grow overnight. The next day cells were spun down at 1800rpm for 3 minutes and resuspended in HM Media (10mM HEPES, 5mM MgSO4, pH 7.2). Solid biotin (Thermo, Waltham, MA) was added to $20 \mu \mathrm{g} / \mathrm{mL}$ for each strain and incubated for 5 minutes with agitation. Cells were diluted with 10 volumes of fresh M1 media before being spun down at $1800 \mathrm{rpm}$ for 3 minutes. After all cells were pelleted, they were washed with fresh M1 media three times. A pre sample was set aside $(100 \mathrm{~mL})$ and the remainder of the cells were resuspended in $4.5 \mathrm{pH} \mathrm{M1}$ media for 45 seconds before being spun down again at 1800rpm for 3 minutes. Cells were then resuspended in $\mathrm{pH} 7.0$ media and allowed to regrow their cilia for 4 hours. A sample was taken pre-biotinylation to use as a control for non-specific streptavidin binding.

Meanwhile, the cilia were isolated from the pre sample. The samples were centrifuged for 3 minutes at $1800 \mathrm{rpm}$. Supernatant was drained and each pellet was resuspended in $2 \mathrm{~mL}$ of $10 \mathrm{mM}$ HEPES ( $\mathrm{pH} 7.4)$. This was repeated 2 times. Then each pellet was resuspended in $1 \mathrm{~mL}$ of fresh ice-cold 4\% HMDS (10mM HEPES pH 7.4, 5mM MgSO4, 1mM DTT, 4\% w/v sucrose). Cells were deciliated by incubating with $25 \mathrm{mM}$ dibucaine for 2 minutes. Then ice cold HMDS with $0.5 \mathrm{mM}$ EGTA was added $(1 \mathrm{~mL}$ per $1.5 \mathrm{~mL}$ of cells). This was then centrifuged for 3 minutes at $1800 \mathrm{rpm}$. Supernatant was collected for each sample. Then HMDS with $25 \%$ sucrose was layered beneath the supernatant $(2 \mathrm{~mL}$ of $25 \%$ HMDS for $1 \mathrm{~mL}$ of supernatant) to create an interface. This was centrifuged at $4^{\circ}$ Celsius for $10 \mathrm{~min}$ at $2400 \mathrm{rpm}$ with no brake to avoid disrupting interface where cilia should now be located. Cilia were removed, pelleted at 21130xg for 30 minutes, then resuspended in lysis buffer (5\% glycerol, 1\% NP-40, 1mM DTT, 1X protease inhibitors). This was repeated with the post samples 4 hours following deciliation. An equal amount of protein was loaded to a 10\% SDS-PAGE gel. The resulting gel was transferred to membrane. The membrane was washed $2 x$ with PBSAT (PBST $+0.1 \%$ BSA), then incubated with HRP-conjugated streptavidin (Thermo, Waltham, MA) for 1 hour. The membrane was then washed 3 times with PBSAT (10 minutes each) and 3 times with PBST (15 minutes each). 
Membrane was probed with West Pico Chemiluminescent Pico Substrate (Invitrogen, Carlsbad, $\mathrm{CA})$.

Homology modeling and sequence studies:

Arp2/3 homology model was created using the Modeller plugin in UCSF Chimera. The template used was 1 U2Z (Nolen, Littlefield, and Pollard 2004; Sali and Blundell 1993; Pettersen et al. 2004). Percent identity and similarity is calculated in relation to the human Arp $2 / 3$ complex members using a MUSCLE alignment in Geneious. The homology model was visualized and conservation was mapped on the protein surface using Chimera (Pettersen et al. 2004).

Statistical analysis:

Statistical analyses were done if GraphPad Prism Version 9. For any experiments comparing 2 groups (Figure 1B, 3D, 4C, 5C, and 5E) an unpaired student's t-test was used to determine $P$ value. For experiments comparing multiple samples at a single time point (Figure 1A and 2B), an ANOVA was used. Finally, for any graphs covering several time points (Figure 1C, 1D, 2C, 3B, and 6E), multiple comparisons were performed (Tukey's and Sidak's). For all experiments ${ }^{* * * *} \mathrm{P}<0.0001,{ }^{* * *} \mathrm{P}<0.001,{ }^{* *} \mathrm{P}<0.01,{ }^{*} \mathrm{P}<0.1$.

\section{ACKNOWLEDGEMENTS:}

Our most sincere gratitude to William Dentler for providing his expertise especially in looking at the electron microscopy images and for his helpful advice, William Snell for generously providing the SAG1-HA strain, Masayuki Onishi for generously providing the nap1 mutant strain, Henry Higgs for his feedback on the manuscript, Ann Lavanway for assistance with microscopy, and the Avasthi lab for all their help throughout the project. We would also like to thank David Sept and Courtney M Schroeder for the help with the original version of this paper and for providing helpful comments throughout the process.

We also thank our funding sources including the Madison and Lila Self Graduate Fellowship at the University of Kansas Medical Center and the MIRA (R35GM128702). Finally, we thank the BioMT core at Dartmouth College (NIH/NIGMS COBRE award P20-GM113132), the Genomics and Molecular Biology Shared Resources Core (NCl Cancer Center Support Grant 5P30CA023108-37), and the KIDDRC NIH U54 HD 090216 at the University of Kansas Medical Center, Kansas City, KS 66160.

\section{REFERENCES:}

Adams, A E, and J R Pringle. 1984. "Relationship of Actin and Tubulin Distribution to Bud Growth in Wild-Type and Morphogenetic-Mutant Saccharomyces Cerevisiae." Journal of Cell Biology 98 (3): 934-45. https://doi.org/10.1083/jcb.98.3.934.

Aghamohammadzadeh, Soheil, and Kathryn R. Ayscough. 2009. "Differential Requirements for Actin during Yeast and Mammalian Endocytosis." Nature Cell Biology 11 (8): 1039-42. https://doi.org/10.1038/ncb1918.

Avasthi, Prachee, Masayuki Onishi, Joel Karpiak, Ryosuke Yamamoto, Luke Mackinder, Martin C Jonikas, Winfield S Sale, Brian Shoichet, John R Pringle, and Wallace F Marshall. 2014. "Actin Is Required for IFT Regulation in Chlamydomonas Reinhardtii." Current Biology 24 (17): 2025-32. https://doi.org/10.1016/j.cub.2014.07.038.

Ayscough, K. R., J. Stryker, N. Pokala, M. Sanders, P. Crews, and D. G. Drubin. 1997. "High Rates of Actin Filament Turnover in Budding Yeast and Roles for Actin in Establishment and 
Maintenance of Cell Polarity Revealed Using the Actin Inhibitor Latrunculin-A." The Journal of Cell Biology 137 (2): 399-416. https://doi.org/10.1083/jcb.137.2.399.

Basu, Roshni, Emilia Laura Munteanu, and Fred Chang. 2014. "Role of Turgor Pressure in Endocytosis in Fission Yeast." Molecular Biology of the Cell 25 (5): 679-87. https://doi.org/10.1091/mbc.E13-10-0618.

Belzile, Olivier, Carmen I Hernandez-Lara, Qian Wang, and William J Snell. 2013. "Regulated Membrane Protein Entry into Flagella Is Facilitated by Cytoplasmic Microtubules and Does Not Require IFT." Current Biology : CB 23 (15): 1460-65. https://doi.org/10.1016/j.cub.2013.06.025.

Bouck, G. Benjamin. 1971. "THE STRUCTURE, ORIGIN, ISOLATION, AND COMPOSITION OF THE TUBULAR MASTIGONEMES OF THE OCHROMONAS FLAGELLUM." Journal of Cell Biology 50 (2): 362-84. https://doi.org/10.1083/jcb.50.2.362.

Campellone, K., and M. Welch. 2010. "A Nucleator Arms Race: Cellular Control of Actin Assembly." Nature Reviews Molecular Cell Biology 11: 237-51.

Carlsson, Anders E., and Philip V. Bayly. 2014. "Force Generation by Endocytic Actin Patches in Budding Yeast." Biophysical Journal 106 (8): 1596-1606. https://doi.org/10.1016/j.bpj.2014.02.035.

Cheng, Xi, Gai Liu, Wenting Ke, Lijuan Zhao, Bo Lv, Xiaocui Ma, Nannan Xu, et al. 2017. “Building a Multipurpose Insertional Mutant Library for Forward and Reverse Genetics in Chlamydomonas." Plant Methods 13 (1): 36. https://doi.org/10.1186/s13007-017-01835.

Craig, Evan W., David M. Mueller, Brae M. Bigge, Miroslava Schaffer, Benjamin D. Engel, and Prachee Avasthi. 2019. "The Elusive Actin Cytoskeleton of a Green Alga Expressing Both Conventional and Divergent Actins." Molecular Biology of the Cell, mbc.E19-03-0141. https://doi.org/10.1091/mbc.E19-03-0141.

Dentler, W L, and C Adams. 1992. "Flagellar Microtubule Dynamics in Chlamydomonas: Cytochalasin D Induces Periods of Microtubule Shortening and Elongation; and Colchicine Induces Disassembly of the Distal, but Not Proximal, Half of the Flagellum." The Journal of Cell Biology 117 (6): 1289-98. https://doi.org/10.1083/jcb.117.6.1289. Dentler, William. 2013. "A Role for the Membrane in Regulating Chlamydomonas Flagellar Length." PLOS ONE 8 (1): e53366. https://doi.org/10.1371/journal.pone.0053366.

Diener, Dennis R, Pietro Lupetti, and Joel L Rosenbaum. 2015. "Proteomic Analysis of Isolated Ciliary Transition Zones Reveals the Presence of ESCRT Proteins." Current Biology : CB 25 (3): 379-84. https://doi.org/10.1016/j.cub.2014.11.066.

Farina, Francesca, Jérémie Gaillard, Christophe Guérin, Yohann Couté, James Sillibourne, Laurent Blanchoin, and Manuel Théry. 2016. "The Centrosome Is an Actin-Organizing Centre." Nature Cell Biology 18 (1): 65-75. https://doi.org/10.1038/ncb3285.

Goode, Bruce L, Julian A Eskin, and Beverly Wendland. 2015. "Actin and Endocytosis in Budding Yeast." Genetics 199 (2): 315-58. https://doi.org/10.1534/genetics.112.145540.

Gournier, Helene, Erin D. Goley, Hanspeter Niederstrasser, Thong Trinh, and Matthew D. Welch. 2001. "Reconstitution of Human Arp2/3 Complex Reveals Critical Roles of Individual Subunits in Complex Structure and Activity." Molecular Cell 8 (5): 1041-52. https://doi.org/10.1016/S1097-2765(01)00393-8. 
Hetrick, Byron, Min Suk Han, Luke A Helgeson, and Brad J Nolen. 2013. "Small Molecules CK-666 and CK-869 Inhibit Actin-Related Protein 2/3 Complex by Blocking an Activating Conformational Change." Chemistry \& Biology 20 (5): 701-12. https://doi.org/10.1016/j.chembiol.2013.03.019.

Hirono, Masafumi, Satomi Uryu, Akio Ohara, Takako Kato-Minoura, and Ritsu Kamiya. 2003. "Expression of Conventional and Unconventional Actins in Chlamydomonas Reinhardtii upon Deflagellation and Sexual Adhesion." Eukaryotic Cell 2 (3): 486-93. https://doi.org/10.1128/ec.2.3.486-493.2003.

Huang, Kaiyao, Dennis R. Diener, Aaron Mitchell, Gregory J. Pazour, George B. Witman, and Joel L. Rosenbaum. 2007. "Function and Dynamics of PKD2 in Chlamydomonas Reinhardtii Flagella." Journal of Cell Biology 179 (3): 501-14. https://doi.org/10.1083/jcb.200704069.

Hunnicutt, G. R., M. G. Kosfiszer, and W. J. Snell. 1990. “Cell Body and Flagellar Agglutinins in Chlamydomonas Reinhardtii: The Cell Body Plasma Membrane Is a Reservoir for Agglutinins Whose Migration to the Flagella Is Regulated by a Functional Barrier." The Journal of Cell Biology 111 (4): 1605-16. https://doi.org/10.1083/jcb.111.4.1605.

Inoue, Daisuke, Dorian Obino, Judith Pineau, Francesca Farina, Jérémie Gaillard, Christophe Guerin, Laurent Blanchoin, Ana-Maria Lennon-Duménil, and Manuel Théry. 2019. “Actin Filaments Regulate Microtubule Growth at the Centrosome." The EMBO Journal 38 (11). https://doi.org/10.15252/embj.201899630.

Jack, Brittany, David M. Mueller, Ann C. Fee, Ashley L. Tetlow, and Prachee Avasthi. 2019. "Partially Redundant Actin Genes in Chlamydomonas Control Transition Zone Organization and Flagellum-Directed Traffic." Cell Reports 27 (8): 2459-2467.e3. https://doi.org/10.1016/j.celrep.2019.04.087.

Jin, Hua, Susan Roehl White, Toshinobu Shida, Stefan Schulz, Mike Aguiar, Steven P. Gygi, J. Fernando Bazan, and Maxence V. Nachury. 2010. "The Conserved Bardet-Biedl Syndrome Proteins Assemble a Coat That Traffics Membrane Proteins to Cilia." Cell 141 (7): 1208-19. https://doi.org/10.1016/j.cell.2010.05.015.

Kaplan, Oktay I., David B. Doroquez, Sebiha Cevik, Rachel V. Bowie, Lara Clarke, Anna A.W.M. Sanders, Katarzyna Kida, Joshua Z. Rappoport, Piali Sengupta, and Oliver E. Blacque. 2012. "Endocytosis Genes Facilitate Protein and Membrane Transport in C. Elegans Sensory Cilia." Current Biology 22 (6): 451-60. https://doi.org/10.1016/j.cub.2012.01.060.

Kato-Minoura, T, S Uryu, M Hirono, and R Kamiya. 1998. "Highly Divergent Actin Expressed in a Chlamydomonas Mutant Lacking the Conventional Actin Gene." Biochemical and Biophysical Research Communications 251 (1): 71-76. https://doi.org/10.1006/bbrc.1998.9373.

Kiesel, Petra, Gonzalo Alvarez Viar, Nikolai Tsoy, Riccardo Maraspini, Peter Gorilak, Vladimir Varga, Alf Honigmann, and Gaia Pigino. 2020. "The Molecular Structure of Mammalian Primary Cilia Revealed by Cryo-Electron Tomography." Nature Structural \& Molecular Biology, September. https://doi.org/10.1038/s41594-020-0507-4.

Kim, Joon, Ji Eun Lee, Susanne Heynen-Genel, Eigo Suyama, Keiichiro Ono, Kiyoung Lee, Trey Ideker, Pedro Aza-Blanc, and Joseph G. Gleeson. 2010. “Functional Genomic Screen for 
Modulators of Ciliogenesis and Cilium Length." Nature 464 (7291): 1048-51. https://doi.org/10.1038/nature08895.

Langousis, Gerasimos, Michelle M. Shimogawa, Edwin A. Saada, Ajay A. Vashisht, Roberto Spreafico, Andrew R. Nager, William D. Barshop, Maxence V. Nachury, James A. Wohlschlegel, and Kent L. Hill. 2016. "Loss of the BBSome Perturbs Endocytic Trafficking and Disrupts Virulence of Trypanosoma Brucei." Proceedings of the National Academy of Sciences of the United States of America 113 (3): 632-37. https://doi.org/10.1073/pnas.1518079113.

Lefebvre, P. A., S. A. Nordstrom, J. E. Moulder, and J. L. Rosenbaum. 1978. “Flagellar Elongation and Shortening in Chlamydomonas. IV. Effects of Flagellar Detachment, Regeneration, and Resorption on the Induction of Flagellar Protein Synthesis." The Journal of Cell Biology 78 (1): 8-27. https://doi.org/10.1083/jcb.78.1.8.

Lefebvre, Paul A. 1995. "Flagellar Amputation and Regeneration in Chlamydomonas." In Methods in Cell Biology, 47:3-7. Elsevier.

Li, Xiaobo, Weronika Patena, Friedrich Fauser, Robert E. Jinkerson, Shai Saroussi, Moritz T. Meyer, Nina Ivanova, et al. 2019. "A Genome-Wide Algal Mutant Library and Functional Screen Identifies Genes Required for Eukaryotic Photosynthesis." Nature Genetics 51 (4): 627-35. https://doi.org/10.1038/s41588-019-0370-6.

Loeffelholz, Ottilie von, Andrew Purkiss, Luyan Cao, Svend Kjaer, Naoko Kogata, Guillaume Romet-Lemonne, Michael Way, and Carolyn A. Moores. 2020. "Cryo-EM of Human Arp2/3 Complexes Provides Structural Insights into Actin Nucleation Modulation by ARPC5 Isoforms." BioRxiv, January, 2020.05.01.071704. https://doi.org/10.1101/2020.05.01.071704.

Marszalek, Joseph R, Xinran Liu, Elizabeth A Roberts, Daniel Chui, Jamey D Marth, David S Williams, and Lawrence S.B Goldstein. 2000. "Genetic Evidence for Selective Transport of Opsin and Arrestin by Kinesin-II in Mammalian Photoreceptors." Cell 102 (2): 175-87. https://doi.org/10.1016/S0092-8674(00)00023-4.

Milenkovic, Ljiljana, Matthew P. Scott, and Rajat Rohatgi. 2009. "Lateral Transport of Smoothened from the Plasma Membrane to the Membrane of the Cilium." The Journal of Cell Biology 187 (3): 365-74. https://doi.org/10.1083/jcb.200907126.

Molla-Herman, Anahi, Rania Ghossoub, Thierry Blisnick, Alice Meunier, Catherine Serres, Flora Silbermann, Chris Emmerson, et al. 2010. "The Ciliary Pocket: An Endocytic Membrane Domain at the Base of Primary and Motile Cilia." Journal of Cell Science 123 (Pt 10): 1785-95. https://doi.org/10.1242/jcs.059519.

Nachury, Maxence V., Alexander V. Loktev, Qihong Zhang, Christopher J. Westlake, Johan Peränen, Andreas Merdes, Diane C. Slusarski, et al. 2007. “A Core Complex of BBS Proteins Cooperates with the GTPase Rab8 to Promote Ciliary Membrane Biogenesis." Cell 129 (6): 1201-13. https://doi.org/10.1016/j.cell.2007.03.053.

Nachury, Maxence V., E. Scott Seeley, and Hua Jin. 2010. "Trafficking to the Ciliary Membrane: How to Get across the Periciliary Diffusion Barrier?" Annual Review of Cell and Developmental Biology 26: 59-87. https://doi.org/10.1146/annurev.cellbio.042308.113337.

Nolen, Brad J., Ryan S. Littlefield, and Thomas D. Pollard. 2004. "Crystal Structures of ActinRelated Protein 2/3 Complex with Bound ATP or ADP." Proceedings of the National 
Academy of Sciences of the United States of America 101 (44): 15627. https://doi.org/10.1073/pnas.0407149101.

Onishi, M., K. Pecani, T. th Jones, J. R. Pringle, and F. R. Cross. 2018. "F-Actin Homeostasis through Transcriptional Regulation and Proteasome-Mediated Proteolysis." Proc Natl Acad Sci U S A 115 (28): E6487-e6496. https://doi.org/10.1073/pnas.1721935115.

Onishi, M., J. R. Pringle, and F. R. Cross. 2016. "Evidence That an Unconventional Actin Can Provide Essential F-Actin Function and That a Surveillance System Monitors F-Actin Integrity in Chlamydomonas." Genetics 202 (3): 977-96. https://doi.org/10.1534/genetics.115.184663.

Onishi, Masayuki, James G. Umen, Frederick R. Cross, and John R. Pringle. 2019. "CleavageFurrow Formation without F-Actin in <em >Chlamydomonas</Em>." BioRxiv, January, 789016. https://doi.org/10.1101/789016.

Papermaster, D. S., B. G. Schneider, and J. C. Besharse. 1985. "Vesicular Transport of Newly Synthesized Opsin from the Golgi Apparatus toward the Rod Outer Segment. Ultrastructural Immunocytochemical and Autoradiographic Evidence in Xenopus Retinas." Investigative Ophthalmology \& Visual Science 26 (10): 1386-1404.

Park, Tae Joo, Brian J. Mitchell, Philip B. Abitua, Chris Kintner, and John B. Wallingford. 2008. "Dishevelled Controls Apical Docking and Planar Polarization of Basal Bodies in Ciliated Epithelial Cells." Nature Genetics 40 (7): 871-79. https://doi.org/10.1038/ng.104.

Pasquale, S M, and U W Goodenough. 1987. "Cyclic AMP Functions as a Primary Sexual Signal in Gametes of Chlamydomonas Reinhardtii." The Journal of Cell Biology 105 (5): 2279-92. https://doi.org/10.1083/jcb.105.5.2279.

Pazour, Gregory J., Sheila A. Baker, James A. Deane, Douglas G. Cole, Bethany L. Dickert, Joel L. Rosenbaum, George B. Witman, and Joseph C. Besharse. 2002. "The Intraflagellar Transport Protein, IFT88, Is Essential for Vertebrate Photoreceptor Assembly and Maintenance." Journal of Cell Biology 157 (1): 103-14. https://doi.org/10.1083/jcb.200107108.

Pedersen, Lotte B., and Joel L. Rosenbaum. 2008. "Intraflagellar Transport (IFT) Role in Ciliary Assembly, Resorption and Signalling." Current Topics in Developmental Biology 85: 2361. https://doi.org/10.1016/S0070-2153(08)00802-8.

Pettersen, Eric F., Thomas D. Goddard, Conrad C. Huang, Gregory S. Couch, Daniel M. Greenblatt, Elaine C. Meng, and Thomas E. Ferrin. 2004. "UCSF Chimera--a Visualization System for Exploratory Research and Analysis." Journal of Computational Chemistry 25 (13): 1605-12. https://doi.org/10.1002/jcc.20084.

Robinson, Robert C., Kirsi Turbedsky, Donald A. Kaiser, Jean-Baptiste Marchand, Henry N. Higgs, Senyon Choe, and Thomas D. Pollard. 2001. "Crystal Structure of Arp2/3 Complex." Science 294 (5547): 1679. https://doi.org/10.1126/science.1066333.

Rohatgi, Rajat, and William J Snell. 2010. "The Ciliary Membrane." Current Opinion in Cell Biology 22 (4): 541-46. https://doi.org/10.1016/j.ceb.2010.03.010.

Rosenbaum, J. L., J. E. Moulder, and D. L. Ringo. 1969. "Flagellar Elongation and Shortening in Chlamydomonas. The Use of Cycloheximide and Colchicine to Study the Synthesis and Assembly of Flagellar Proteins." The Journal of Cell Biology 41 (2): 600-619. https://doi.org/10.1083/jcb.41.2.600. 
Saito, Masaki, Wataru Otsu, Kuo-Shun Hsu, Jen-Zen Chuang, Teruyuki Yanagisawa, Vincent Shieh, Taku Kaitsuka, Fan-Yan Wei, Kazuhito Tomizawa, and Ching-Hwa Sung. 2017. "Tctex-1 Controls Ciliary Resorption by Regulating Branched Actin Polymerization and Endocytosis." EMBO Reports 18 (8): 1460-72. https://doi.org/10.15252/embr.201744204.

Sali, A., and T. L. Blundell. 1993. "Comparative Protein Modelling by Satisfaction of Spatial Restraints." Journal of Molecular Biology 234 (3): 779-815. https://doi.org/10.1006/jmbi.1993.1626.

Spector, Ilan, Nava R. Shochet, Dina Blasberger, and Yoel Kashman. 1989. "Latrunculins-Novel Marine Macrolides That Disrupt Microfilament Organization and Affect Cell Growth: I. Comparison with Cytochalasin D." Cell Motility 13 (3): 127-44. https://doi.org/10.1002/cm.970130302.

Wingfield, Jenna L, Ilaria Mengoni, Heather Bomberger, Yu-Yang Jiang, Jonathon D Walsh, Jason M Brown, Tyler Picariello, et al. 2017. "IFT Trains in Different Stages of Assembly Queue at the Ciliary Base for Consecutive Release into the Cilium." Edited by Erwin J G Peterman. ELife 6 (May): e26609. https://doi.org/10.7554/eLife.26609.

Winter, D. C., E. Y. Choe, and R. Li. 1999. "Genetic Dissection of the Budding Yeast Arp2/3 Complex: A Comparison of the in Vivo and Structural Roles of Individual Subunits." Proceedings of the National Academy of Sciences of the United States of America 96 (13): 7288-93. https://doi.org/10.1073/pnas.96.13.7288.

Wood, Christopher R., and Joel L. Rosenbaum. 2014. "Proteins of the Ciliary Axoneme Are Found on Cytoplasmic Membrane Vesicles during Growth of Cilia." Current Biology 24 (10): 1114-20. https://doi.org/10.1016/j.cub.2014.03.047.

Wu, Chien-Ting, Hsin-Yi Chen, and Tang K. Tang. 2018. "Myosin-Va Is Required for Preciliary Vesicle Transportation to the Mother Centriole during Ciliogenesis." Nature Cell Biology 20 (2): 175-85. https://doi.org/10.1038/s41556-017-0018-7.

Zuo, Xiaofeng, Wei Guo, and Joshua H. Lipschutz. 2009. "The Exocyst Protein Sec10 Is Necessary for Primary Ciliogenesis and Cystogenesis In Vitro." Molecular Biology of the Cell 20 (10): 2522-29. https://doi.org/10.1091/mbc.e08-07-0772. 